This article may be downloaded for personal use only. Any other use requires prior permission of the author and AIP Publishing. This article appeared in J. Chem. Phys. 152, 034202 (2020) and may be found at https://doi.org/10.1063/1.5133408

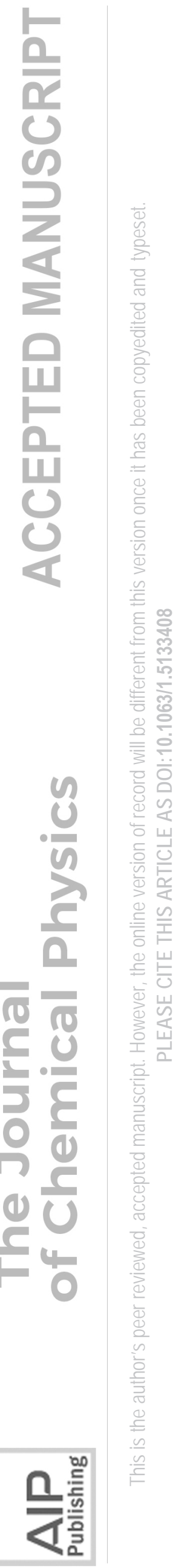

\title{
Optically-generated Overhauser Dynamic Nuclear Polarization: A Numerical Analysis
}

Daniel J. Cheney ${ }^{1}$ and Christopher J. Wedge ${ }^{1, a}$

${ }^{1}$ Department of Chemical Sciences, University of Huddersfield, Queensgate, Huddersfield, UK.

Recently, an alternative approach to dynamic nuclear polarization (DNP) in the liquid state was introduced, using optical illumination instead of microwave pumping. By exciting a suitable dye to the triplet state which undergoes diffusive encounter with a persistent radical forming a quartet-doublet pair in the encounter complex, dynamic electron polarization (DEP) is generated via the radical-triplet pair mechanism (RTPM). Subsequent cross-relaxation generates nuclear polarization without the need for microwave saturation of the electronic transitions. Here, we present a theoretical justification for the initial experimental results, by means of numerical simulations. These allow investigation of the effects of various experimental parameters, such as radical and dye concentrations, sample geometry, and laser power, on the DNP enhancement factors, providing targets for experimental optimization. It is predicted that reducing the sample volume will result in larger enhancements, by permitting a higher concentration of triplets in a sample of increased optical density. We also explore the effects of pulsed laser rather than continuous-wave illumination, rationalising the failure to observe the optical DNP effect under illumination conditions common to DEP experiments. Examining the influence of illumination duty cycle the conditions necessary to permit use of pulsed illumination without compromising signal enhancement are determined, which may reduce undesirable laser heating effects. This first simulation of the optical DNP method therefore underpins the further development of the technology.

\section{INTRODUCTION}

Among all of the hyperpolarization techniques for overcoming the intrinsically low sensitivity of Nuclear Magnetic Resonance (NMR) spectroscopy, Dynamic Nuclear Polarization (DNP) is the most studied and widely applicable. ${ }^{1}$ It was first proposed by Overhauser in $1953,{ }^{2}$ and demonstrated experimentally by Carver and Slichter, ${ }^{3}$ that saturating Electron Paramagnetic Resonance (EPR) transitions results in an increase in NMR sensitivity. This effect, due to cross-relaxation between the electron and nuclear spins, was later extended from lithium to free radicals in solution, ${ }^{4}$ but has never been widely applied due to technical challenges and problems with reducing Overhauser efficiency at the high magnetic fields necessary for high resolution NMR spectroscopy. Although DNP in the solid state ${ }^{5}$ has attracted a significant amount of interest since its renaissance in the 1990s with gyrotron driven systems now commercially available, progress with

\footnotetext{
${ }^{\text {a }}$ Author to whom correspondence should be addressed. Electronic address: c.wedge@ hud.ac.uk
} 
This article may be downloaded for personal use only. Any other use requires prior permission of the author and AIP Publishing. This article appeared in J. Chem. Phys. 152, 034202 (2020) and may be found at https://doi.org/10.1063/1.5133408

solution DNP has been rather slower. ${ }^{67}$ Solution studies have mainly focussed on water, since its small size leads to fast rotational dynamics, leading to relatively high enhancements even at high magnetic fields. ${ }^{7-12}$ There is great potential for the study of hydration dynamics of proteins and soft materials, ${ }^{13,14}$ as well as polarization transfer to other molecules. ${ }^{15}$ However, since water interacts very strongly with the electric fields of microwaves, dielectric heating is a significant issue in aqueous solutions, particularly when working with temperature-sensitive biological molecules, or when the coupling factor needs to be known accurately. ${ }^{16}$

Recently, we showed that instead of using microwaves, DNP could be achieved by using optical pumping to generate Chemically-Induced Dynamic Electron Polarization (CIDEP). ${ }^{17}$ Utilizing the radical-triplet pair mechanism (RTPM) which is able to polarize a persistent radical through interaction with a photogenerated triplet state, leads to non-Boltzmann electron spin polarization that by slow cross-relaxation can be transferred to nuclei without any competition with a radical decay process. This not only has the potential to overcome the theoretical limit for DNP enhancements based on the ratio of the Boltzmann electronic and nuclear polarizations, but also avoids the technically demanding microwave irradiation step. The proof of principle experiment for this approach used the radical-dye pair $(2,2,6,6)$-tetramethylpiperidin-1-yl)oxyl (TEMPO) and rose bengal, which in an extensive study into the quenching of xanthene dyes in aqueous solution had previously been determined to produce a very strong CIDEP effect. ${ }^{18}$ In common to conventional microwave pumped DNP studies the NMR enhancement was initially detected on the solvent signal, with ${ }^{1} \mathrm{H}$ enhancements of around -3 observed in aqueous solution at low field $(0.342 \mathrm{~T})$ simply by applying continuous optical illumination (Figure 1(a)). ${ }^{17}$ The enhancement was increased to around -4 when using $80(\mathrm{v} / \mathrm{v}) \% \mathrm{D}_{2} \mathrm{O}$ owing to increased nuclear spin lattice relaxation time, and an optimum radical concentration of $1 \mathrm{mM}$ was found, as shown in Figure 1(b). In conventional microwave pumped DNP the observed enhancement increases with radical concentration, due to the stronger paramagnetic relaxation (Figure 1(c)), a trend also observed to some extent in the present case. At higher radical concentrations however the enhancement decreases, indicating that the fraction of the radicals polarized by the triplets is reduced at high radical concentrations (Figure 1(d)), a problem which may potentially be overcome by tethering the radical and chromophore to remove the reliance on intermolecular quenching. The key result of this initial study was to demonstrate that optically driven Overhauser DNP is possible under conditions of continuous optical illumination, not using the low repetition pulsed illumination familiar to previous CIDEP studies. Building upon this finding a similar optical DNP effect has already been shown in a covalently linked fullerene-nitroxide system, though with an NMR enhancement $<2$ arising from competing effects of emissive and absorptive CIDEP. ${ }^{19}$ 
This article may be downloaded for personal use only. Any other use requires prior permission of the author and AIP Publishing. This article appeared in J. Chem. Phys. 152, 034202 (2020) and may be found at https://doi.org/10.1063/1.5133408

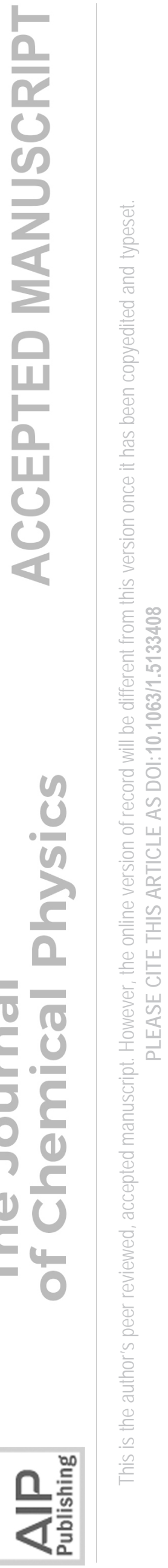

(a)

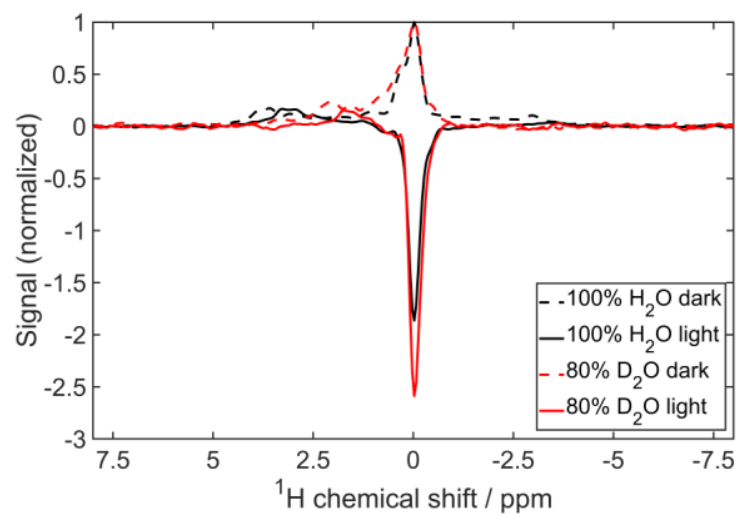

(c)

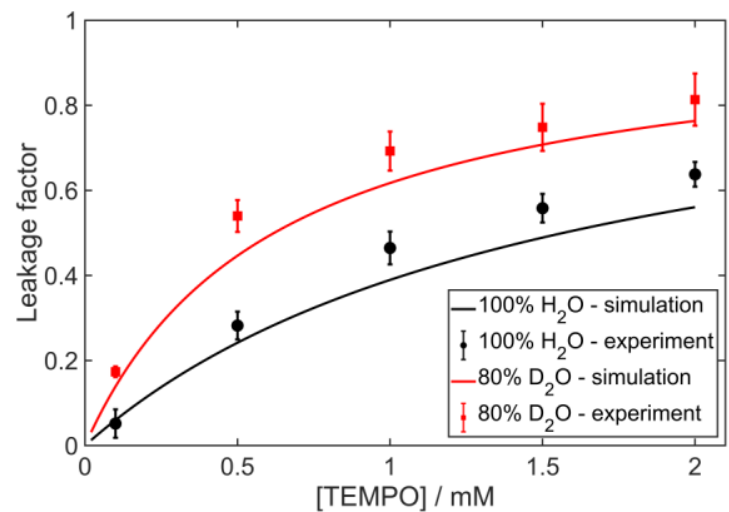

(b)

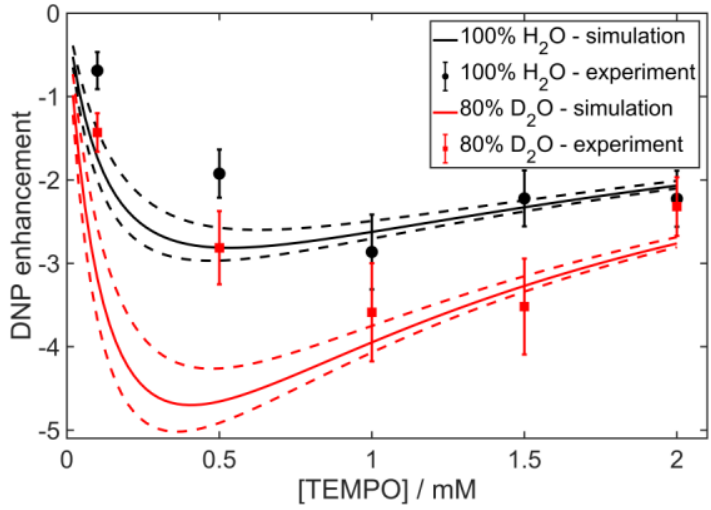

(d)

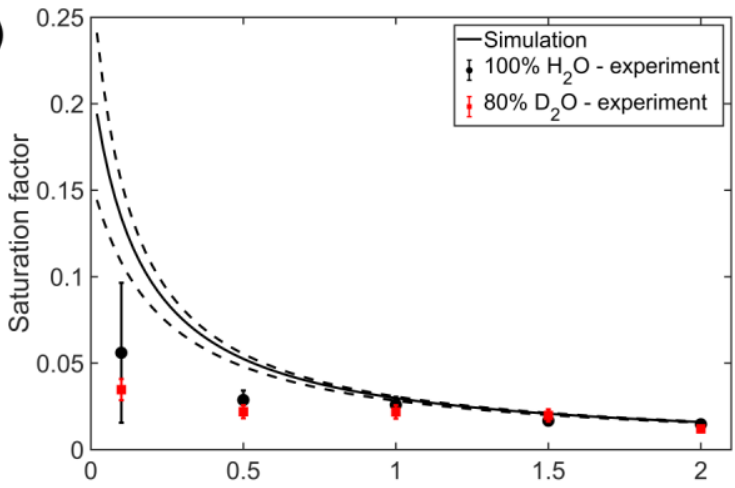

Figure 1: (a) ${ }^{1} \mathrm{H}$ NMR spectra of aqueous solvent at $14.6 \mathrm{MHz}$ in a sample of a $0.2 \mathrm{mM}$ rose bengal and $1.0 \mathrm{mM}$ TEMPO, with and without $1 \mathrm{~W}$ illumination at $520 \mathrm{~nm}$. (b) enhancment factor, (c) leakage factor, and (c) saturation factor as a function of TEMPO concentration, with rose bengal concentration of $0.2 \mathrm{mM}$ in $\mathrm{H}_{2} \mathrm{O}$ or $20 \% \mathrm{H}_{2} \mathrm{O} / 80 \%$ $\mathrm{D}_{2} \mathrm{O}$ mixture, based on $10 \mathrm{~s}$ illumination time with $10 \mu \mathrm{min}^{-1}$ sample flow. Experimental data are taken from ref. 17, with the simulations of key parameters as described below. The dashed lines in (b) and (d) refer to the upper and lower bounds of the uncertainty in the measurement of the rate constant for bimolecular triplet quenching by the radical, $k_{\mathrm{q}}$.

While the demonstration of these novel optical hyperpolarization mechanisms is exciting, the enhancements observed thus far are relatively small. To date optical methods have not matched the results of microwave pumping experiments as the departure of the electron spin system from equilibrium, quantified by the electron saturation factor, does not when averaged over all radicals exceed the value of unity achievable by complete microwave saturation of the electronic transition. In optical DNP there are several parameters upon which the saturation factor depends, such as the radical and dye concentrations, laser power, optical path length of the sample, and the identity of the sample itself. Here, we will account for the results on the intermolecular system using numerical simulations combining a Bloch kinetic treatment of the DEP step with analysis of the coupled electron-nuclear spin system, achieving satisfactory agreement with the experimental data available to date. Through exploration of the available parameter space we predict how the NMR signal enhancement may be further optimized; to our knowledge, such studies have not previously been carried out for optically-driven Overhauser DNP. Finally, following Vieth and co-workers, ${ }^{20,21}$ we also model the effects of pulsed illumination to determine optimal illumination schemes and duty cycle, informing future attempts to realize this approach experimentally. 
This article may be downloaded for personal use only. Any other use requires prior permission of the author and AIP Publishing. This article appeared in J. Chem. Phys. 152, 034202 (2020) and may be found at https://doi.org/10.1063/1.5133408

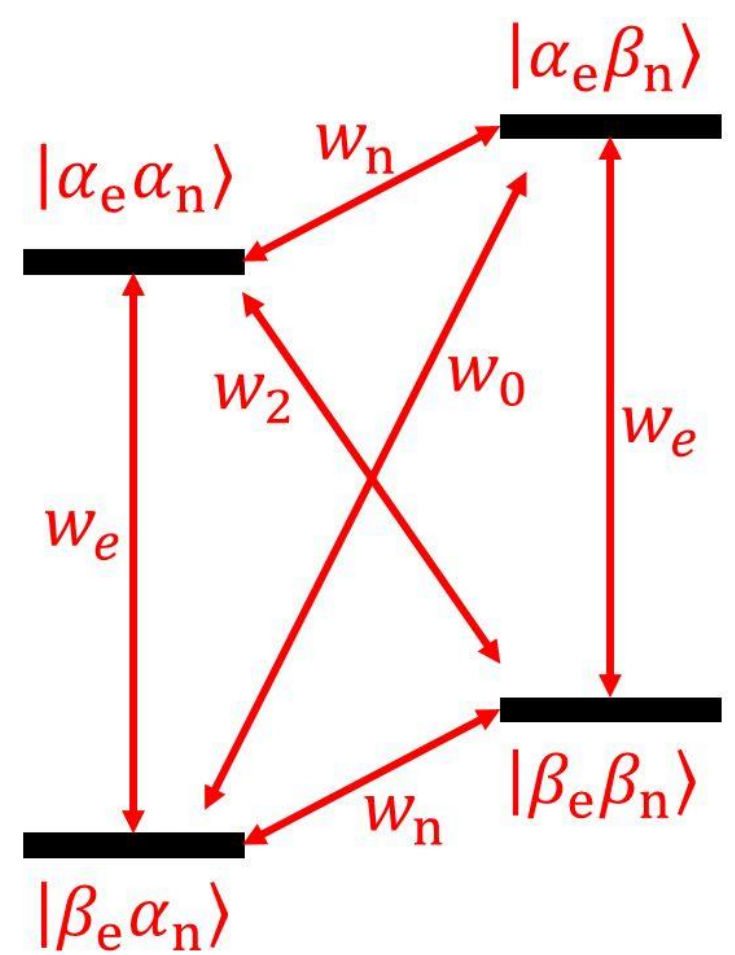

Figure 2: Energy level diagram for a spin-1/2 nucleus hyperfine coupled to an unpaired electron. $w_{0}, w_{\mathrm{e}}, w_{\mathrm{n}}$, and $w_{2}$ refer to zero-quantum, electron and nuclear single-quantum, and double-quantum transitions, respectively.

\section{THEORETICAL BACKGROUND}

\section{A. Overhauser DNP}

A spin-1/2 nucleus hyperfine-coupled to an electron results in the four energy levels, as shown in Figure 2. ${ }^{16,22}$ The Overhauser effect is conventionally driven by the saturation of the EPR transitions $\left(w_{\mathrm{e}}\right)$, which results in polarization transfer to the nuclei owing to disparity between the zero- and double-quantum cross-relaxation terms $\left(w_{0}\right.$ and $\left.w_{2}\right)$. The familiar steady state solution to the problem under continuous pumping is the Overhauser equation for the enhancement factor, $\varepsilon^{2,22,23}$

$$
\varepsilon=\frac{\left\langle I_{\mathrm{z}}\right\rangle-I_{\mathrm{z}}^{0}}{I_{\mathrm{z}}^{0}}=-\xi f s\left|\frac{\gamma_{\mathrm{e}}}{\gamma_{\mathrm{n}}}\right|
$$

where $\xi$ is the coupling factor, $f$ is the leakage factor, $s$ is the saturation factor and $\gamma_{\mathrm{e}, \mathrm{n}}$ are the electron and nuclear gyromagnetic ratios.

The coupling factor may vary between +0.5 (for purely dipolar electron-nuclear interactions) and -1 (for purely scalar interactions), with the former situation being typical for protons. ${ }^{16}$ However, since for efficient polarization transfer the hyperfine interaction must be modulated by motions on a timescale close to the EPR frequency, $\xi$ quickly decreases at high magnetic fields and long correlation times ${ }^{24}$ necessitating polarization at magnetic fields of $<1 \mathrm{~T}$, although significant coupling factors have recently been observed for scalarcoupled ${ }^{13} \mathrm{C}$ at high-field. ${ }^{25,26}$ The leakage factor describes the contribution of paramagnetic relaxation to the total nuclear relaxation: 
This article may be downloaded for personal use only. Any other use requires prior permission of the author and AIP Publishing. This article appeared in J. Chem. Phys. 152, 034202 (2020) and may be found at https://doi.org/10.1063/1.5133408

$$
f=1-\frac{T_{1 \mathrm{n}}}{T_{1 \mathrm{n}}^{0}}
$$

where $T_{1 \mathrm{n}}^{0}$ is the relaxation time in the absence of radical, and the saturation factor describes the degree to which the microwave field drives the electron spins out of equilibrium:

$$
s=1-\frac{S_{\mathrm{z}}}{S_{\mathrm{z}}^{0}}
$$

Since the microwave field cannot invert the populations of the electron spin states, but can only equalize them, the saturation factor has a maximum value of 1 and overall the DNP enhancement may vary between $+0.5\left|\gamma_{\mathrm{e}} / \gamma_{\mathrm{n}}\right|$ and $-\left|\gamma_{\mathrm{e}} / \gamma_{\mathrm{n}}\right|$ (that is the maximum enhancement achievable for proton spins varies between +330 and -660$){ }^{8}{ }^{8,13}$ However, since the EPR transitions are not excited directly in the optical approach population inversion is theoretically possible for the electron spin system, providing $|s|>1$, which if achieved for the majority of radicals will overcome the theoretical limit on the maximum DNP enhancement.

\section{B. Radical-triplet pair mechanism}

As reviewed elsewhere there are numerous CIDEP mechanisms, ${ }^{27,28}$ with the RTPM and related Electron Spin Polarization Transfer (ESPT) mechanisms unique in that they can polarize persistent radicals, as opposed to the triplet mechanism and the radical pair mechanism which polarize transient radicals. For an optical DNP scheme it is essential that the radical persists for sufficient time to permit transfer of polarization to the nuclei, hence the RTPM and ESPT are ideal candidates as there is no radical decay process in competition with polarization transfer. In the ESPT, optical polarization of the triplet is transferred to the radical, demanding slow spin-lattice relaxation in the triplet state, and hence this mechanism is usually applicable only in solids or highly viscous solutions. ${ }^{27,29}$ The RTPM on the other hand can produce electronic hyperpolarization of the radical even on encounter with a thermalized triplet,,$^{30}$ so is active in low viscosity solutions in which transfer of electron polarization to nuclei via the Overhauser mechanism will be most effective.

The key steps of the RTPM are shown diagrammatically in Figure $3 .{ }^{31}$ The variation in energy of the radical-triplet pair states as a function of the intermolecular distance $r$ may be calculated from the radicaltriplet Hamiltonian, ${ }^{32}$

$$
\widehat{H}(r)=g \mu_{B} B_{0}\left(S_{\mathrm{T}_{\mathrm{Z}}}+S_{\mathrm{R}_{\mathrm{Z}}}\right)-\frac{1}{3} J(r)\left(1+4 \boldsymbol{S}_{\mathrm{T}} \cdot \boldsymbol{S}_{\mathrm{R}}\right)+D_{\mathrm{zfs}}\left(S_{T_{\zeta}}^{2}-\frac{1}{3} \boldsymbol{S}_{T}^{2}\right)
$$

in which $\boldsymbol{S}_{\mathrm{R}}$ and $\boldsymbol{S}_{\mathrm{T}}$ represent singlet and triplet spin states and $D_{\mathrm{zfs}}$ is the axial component of the zero-field splitting parameter (the transverse component has been neglected). The second term represents the radical triplet exchange interaction which takes the conventional form,

$$
J(r)=J_{0} \exp \{-\beta(r-d)\}
$$


This article may be downloaded for personal use only. Any other use requires prior permission of the author and AIP Publishing. This article appeared in J. Chem. Phys. 152, 034202 (2020) and may be found at https://doi.org/10.1063/1.5133408

where $J_{0}<0$ is the interaction strength at the distance of closest approach $d$, and $\beta$ a range parameter. For simplicity the g-factors and hence electron Zeeman splitting's of the two species have been assumed to be identical. The radical-triplet encounter complex is seen to consist of doublet and quartet states. The doublets may relax to the ground state (radical-singlet pair) via a spin-allowed process known as enhanced intersystem crossing (EISC), as the radical has increased the triplet decay rate compared to the spinforbidden triplet to singlet transition. As the equivalent process for the quartets remains spin-forbidden these diffusively separate, passing through the crossings of the $\left|Q_{-3 / 2}^{R T}\right\rangle-\left|D_{+1 / 2}^{R T}\right\rangle,\left|Q_{-1 / 2}^{R T}\right\rangle-\left|D_{+1 / 2}^{R T}\right\rangle$, and $\left|Q_{-3 / 2}^{R T}\right\rangle-\left|D_{-1 / 2}^{R T}\right\rangle$ levels to give separated triplet and radical. This process alone appears to have left the radical spin states equally populated, however, because the zero-field splitting of the triplet does not commute with the Hamiltonian of the radical-triplet pair the states are mixed. ${ }^{28}$ This turns the crossings into level anti crossings (LACs), resulting in generation of emissive electron spin polarization in the case of negative exchange interaction. It will be apparent from the diagram that the relative magnitude of the exchange and Zeeman interactions will determine the separation at which the LACs occur, and therefore little polarization will be generated if the applied field is so large that these occur at a distance less than the hard sphere collision radius.

\section{COMPUTATIONAL METHODS}

\section{A. Generation of triplets}

The first stage in the simulation of the spin dynamics of the system is to determine the time dependent concentration of the photoexcited triplet state from which the DEP is generated. The number of triplet molecules generated from a given number of photons, $n_{\text {photon }}$ is:

$$
n_{\mathrm{T}}=n_{\text {photon }} \Phi p_{\mathrm{A}}
$$

where $\Phi$ is the quantum yield for intersystem crossing (ISC), which equals 0.786 for rose bengal in aqueous solution, ${ }^{33}$ and $p_{\mathrm{A}}$ is the probability of a photon being absorbed by the sample. From the Beer-Lambert law:

$$
n_{\mathrm{T}}=n_{\text {photon }} \Phi\left(1-10^{-\varepsilon[\mathrm{S}] l}\right)
$$

Where $\varepsilon$ is the molar extinction coefficient $\left(48000 \mathrm{M}^{-1} \mathrm{~cm}^{-1}\right.$ at $\left.532 \mathrm{~nm}\right),{ }^{17}[\mathrm{~S}]$ is the ground-state dye concentration, and $l$ is the optical path length. The experiments in ref. 17 were carried out in a cylindrical tube of $1.0 \mathrm{~mm}$ inner diameter (I.D.), for which the average path length was calculated to be $0.78 \mathrm{~mm}$. Our simulations will also consider a $0.4 \mathrm{~mm}$ I.D. tube, which has a path length of $0.31 \mathrm{~mm}$. The height of the illumination region was $5.0 \mathrm{~mm}$. The rate of triplet build-up depends on the photon flux and hence illumination power according to:

$$
\frac{d n_{\mathrm{T}}}{d t}=\frac{d n_{\text {photon }}}{d t} \Phi\left(1-10^{-\varepsilon[\mathrm{S}] l}\right)=P \frac{\lambda}{h c} \Phi\left(1-10^{-\epsilon[\mathrm{S}] l}\right)
$$


This article may be downloaded for personal use only. Any other use requires prior permission of the author and AIP Publishing. This article appeared in J. Chem. Phys. 152, 034202 (2020) and may be found at https://doi.org/10.1063/1.5133408

where $P$ is the laser power, $h$ is the Planck constant, $\lambda$ is the wavelength, and $c$ is the speed of light. Converting the number of triplet molecules to a concentration gives:

$$
\frac{d\left[{ }^{3} \mathrm{RB}^{2-}\right]}{d t}=P \frac{\lambda}{h c} \frac{1}{V N_{\mathrm{A}}} \Phi\left(1-10^{-\varepsilon[\mathrm{S}] l}\right)
$$

where $V$ is the sample volume, and $N_{\mathrm{A}}$ is the Avogadro constant.
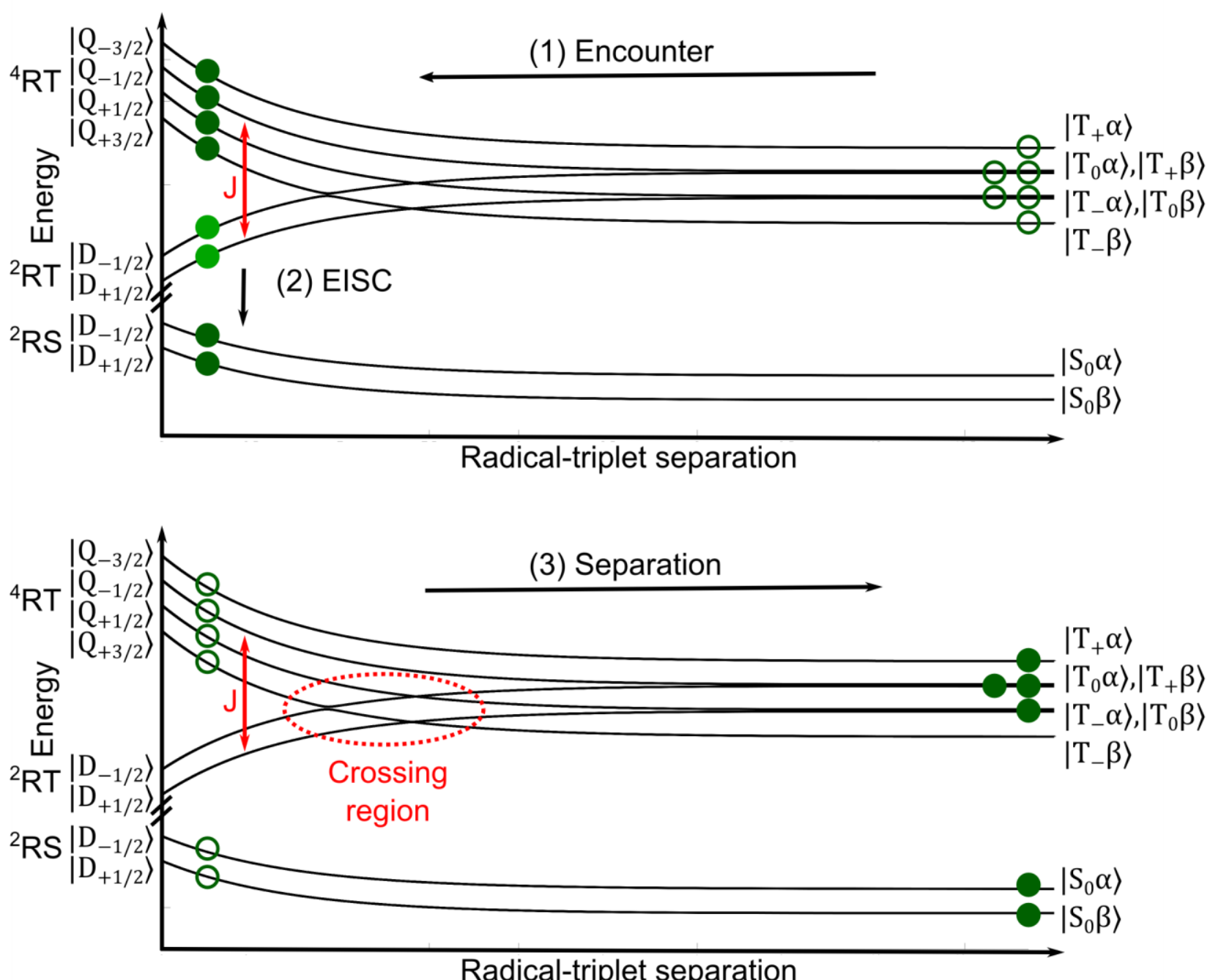

Figure 3: Generation of CIDEP via the RTPM: (1) Initial encounter of the radical and triplet to form a complex; (2) 'enhanced intersystem crossing' permits spin-allowed quenching of the doublet states to the ground-state; (3) separation of the complex with passage through the LAC region to generate electron spin polarization. Energy levels may be calculated from equation 4 with the magnitude of the applied magnetic field $B_{0}$ determining the magnitude of the Zeeman splitting between the energy levels at large separation distances. 
This article may be downloaded for personal use only. Any other use requires prior permission of the author and AIP Publishing. This article appeared in J. Chem. Phys. 152, 034202 (2020) and may be found at https://doi.org/10.1063/1.5133408

\begin{tabular}{|c|c|}
\hline Rate Constant & Value \\
\hline$k_{\mathrm{t}}$ & $(2.14 \pm 0.85) \times 10^{4} \mathrm{~s}^{-1}$ \\
\hline$k_{\mathrm{tt}}$ & $(7.79 \pm 1.35) \times 10^{8} \mathrm{~s}^{-1} \mathrm{M}^{-1}$ \\
\hline$k_{\mathrm{sq}}$ & $(5.37 \pm 1.11) \times 10^{8} \mathrm{~s}^{-1} \mathrm{M}^{-1}$ \\
\hline$k_{\text {redox* }}$ & $(5.39 \pm 1.43) \times 10^{7} \mathrm{~s}^{-1} \mathrm{M}^{-1}$ \\
\hline$k_{\text {redox** }}$ & $(1.73 \pm 0.34) \times 10^{8} \mathrm{~s}^{-1} \mathrm{M}^{-1}$ \\
\hline$k_{\text {-T }}$ & $(4.72 \pm 1.56) \times 10^{9} \mathrm{~s}^{-1} \mathrm{M}^{-1}$ \\
\hline
\end{tabular}

Table 1: Rate constants for the photophysics of rose bengal. All values are taken from Ref. ${ }^{33}$.

The photochemistry of rose bengal was modelled according to a study by Ludvíková et al., ${ }^{33}$ with the following triplet deactivation processes known to occur: ${ }^{34-36}$

$$
\begin{aligned}
& { }^{3} \mathrm{RB}^{2-} \stackrel{k_{\mathrm{t}}}{\rightarrow} \mathrm{RB}^{2-} \\
& { }^{3} \mathrm{RB}^{2-}+{ }^{3} \mathrm{RB}^{2-} \stackrel{k_{\mathrm{tt}}}{\rightarrow} \mathrm{RB}^{2-}+{ }^{1} \mathrm{RB}^{2-*} \\
& { }^{3} \mathrm{RB}^{2-}+{ }^{1} \mathrm{RB}^{2-} \stackrel{k_{\mathrm{sq}}}{\rightarrow} 2^{1} \mathrm{RB}^{2-} \\
& { }^{3} \mathrm{RB}^{2-}+{ }^{1} \mathrm{RB}^{2-} \stackrel{k_{\text {redox* }}}{\longrightarrow} \mathrm{RB}^{\cdot 3-}+\mathrm{RB}^{\cdot-} \\
& { }^{3} \mathrm{RB}^{2-}+{ }^{3} \mathrm{RB}^{2-} \stackrel{k_{\text {redox } * *}}{\longrightarrow} \mathrm{RB}^{\cdot 3-}+\mathrm{RB}^{\cdot-}
\end{aligned}
$$

These correspond to decay of the triplet to the ground state $\left(k_{\mathrm{t}}\right)$, triplet-triplet annihilation $\left(k_{\mathrm{tt}}\right)$, selfquenching by the ground-state $\left(k_{\mathrm{sq}}\right)$, electron exchange with the ground-state $\left.\left(k_{\mathrm{redox}}\right)^{*}\right)$ and disproportionation with another triplet $\left(k_{\mathrm{redox}} *^{*}\right)$, respectively. The oxidized and reduced forms of the dye can undergo back electron transfer to reform the ground-state $(k-\mathrm{eT})$.

$$
\mathrm{RB}^{\cdot 3-}+\mathrm{RB}^{\cdot-} \stackrel{k_{-\mathrm{eT}}}{\longrightarrow} 2^{1} \mathrm{RB}^{2-}
$$

The rate constants for these processes were measured previously using laser flash photolysis, and are given in Table 1. In the presence of a radical, there is of course an additional process, intermolecular quenching of the triplet by the radical which occurs with rate constant, $k_{\mathrm{q}}$ :

$$
{ }^{3} \mathrm{RB}^{2-}+\mathrm{R} \stackrel{k_{\mathrm{q}}}{\rightarrow}{ }^{1} \mathrm{RB}^{2-}+\mathrm{R}^{\#}
$$

where the \# indicates that the radical is spin-polarized. The rate constant for this process was determined through time-resolved EPR (TR-EPR) to be $(9.0 \pm 0.2) \times 10^{8} \mathrm{M}^{-1} \mathrm{~s}^{-1}$ in aqueous solution without $\mathrm{pH}$ buffer. ${ }^{37}$ With knowledge of the rate of triplet generation under continuous wave $(\mathrm{CW})$ irradiation, and the decay pathways, the resultant differential equations are: 
This article may be downloaded for personal use only. Any other use requires prior permission of the author and AIP Publishing. This article appeared in J. Chem. Phys. 152, 034202 (2020) and may be found at https://doi.org/10.1063/1.5133408

$$
\begin{aligned}
& \frac{d\left[{ }^{3} \mathrm{RB}^{2-}\right]}{d t}=\frac{d[\mathrm{~T}]}{d t} \\
& =P \frac{\lambda}{h c} \frac{1}{V N_{\mathrm{A}}} \Phi\left(1-10^{-\epsilon[\mathrm{S}] l}\right)-k_{\mathrm{q}}[\mathrm{R}][\mathrm{T}]-k_{\mathrm{t}}[\mathrm{T}]-(2-\Phi) k_{\mathrm{tt}}[\mathrm{T}]^{2}-k_{\text {redox } *}[\mathrm{~T}][\mathrm{S}] \\
& -2 k_{\text {redox** }}[\mathrm{T}]^{2}-k_{\mathrm{sq}}[\mathrm{T}][\mathrm{S}] \\
& \frac{d\left(\left[\mathrm{RB}^{\cdot 3-}\right]+\left[\mathrm{RB}^{\cdot-}\right]\right)}{d t}=\frac{d[\text { Redox }]}{d t} \\
& =2\left(k_{\text {redox } *}[\mathrm{~T}][\mathrm{S}]+k_{\text {redox } *}[\mathrm{~T}]^{2}-k_{-\mathrm{eT}}[\operatorname{Redox}]^{2}\right) \\
& \frac{d\left[{ }^{1} \mathrm{RB}^{2-}\right]}{d t}=\frac{d[\mathrm{~S}]}{d t} \\
& =k_{\mathrm{t}}[\mathrm{T}]+k_{\mathrm{sq}}[\mathrm{T}][\mathrm{S}]+(2-\Phi) k_{\mathrm{tt}}[\mathrm{T}]^{2}+2 k_{-\mathrm{eT}}[\text { Redox }]^{2}-k_{\text {redox } *}[\mathrm{~T}][\mathrm{S}] \\
& -P \frac{\lambda}{h c} \frac{1}{V N_{\mathrm{A}}} \Phi\left(1-10^{-\epsilon[\mathrm{S}] l}\right)
\end{aligned}
$$

where for brevity symbols $[\mathrm{S}]$ and $[\mathrm{T}]$ have been substituted for $\left[{ }^{3} \mathrm{RB}^{2-}\right]$ and $\left[{ }^{1} \mathrm{RB}^{2-}\right]$, and [Redox] for the sum $\left(\left[\mathrm{RB}^{\cdot 3-}\right]+\left[\mathrm{RB}^{\cdot-}\right]\right)$ as this pair of redox states are always created and consumed together. Note that the factor of $(2-\Phi)$ in the triplet-triplet annihilation term arises from the fact that this process generates a ground-state singlet and an excited singlet, the latter of which can undergo ISC to regenerate a triplet. ${ }^{33}$

\section{B. Electron and nuclear magnetization}

The electron and nuclear magnetizations are propagated as:

$$
\begin{aligned}
& \frac{d\left\langle S_{\mathrm{z}}\right\rangle}{d t}=P_{\mathrm{n}} k_{\mathrm{q}}[\mathrm{R}][\mathrm{T}]-\frac{\left\langle S_{\mathrm{z}}\right\rangle-S_{\mathrm{z}}^{0}}{T_{1 \mathrm{e}}} \\
& \frac{d\left\langle I_{\mathrm{z}}\right\rangle}{d T}=-\left(w_{2}-w_{0}\right)\left(\left\langle S_{\mathrm{z}}\right\rangle-S_{\mathrm{z}}^{0}\right)-\left(w_{0}+2 w_{\mathrm{n}}+w_{2}+\frac{1}{T_{1 \mathrm{n}}^{0}}\right)\left(\left\langle I_{\mathrm{z}}\right\rangle-I_{\mathrm{z}}^{0}\right)
\end{aligned}
$$

where $P_{\mathrm{n}}$ is the net CIDEP generated per quenching event, and $T_{1 \mathrm{e}}$ the electron spin lattice relaxation time. By EPR and NMR inversion recovery experiments $T_{1 \mathrm{e}}$ was found to be $623 \mathrm{~ns}$, and $T_{1 \mathrm{n}}$ was $2.587 \mathrm{~s}$ and 6.542 s in $100 \% \mathrm{H}_{2} \mathrm{O}$ and 1:4 $\mathrm{H}_{2} \mathrm{O}: \mathrm{D}_{2} \mathrm{O}$ respectively, however in this case the individual transition probabilities are required as described below. The polarization generated per quenching event, $P_{\mathrm{n}}$, may be obtained experimentally through numerical fitting of time-resolved EPR data by equation 20, subject to complete and accurate knowledge of the photochemical system as described above. Alternatively the value may be calculated using an equation derived by Shushin for the strong coupling limit $\left(\left|J_{0}\right|>\omega_{0}\right)^{32}$

$$
\left|P_{\mathrm{n}}\right|=\frac{\pi}{45} \frac{D_{\mathrm{zfs}}^{2}}{\omega_{0}} \frac{d}{\beta D_{\mathrm{rt}}}\left(\frac{1}{1+x^{2}}+\frac{4}{4+x^{2}}\right)
$$

where the exchange interaction is assumed to take the form of equation $5, D_{\mathrm{rt}}$ is the translational diffusion coefficient of the radical-triplet pair and $x=1 /\left(\omega_{0} \tau_{c}\right)$, with $\tau_{\mathrm{c}}$ the rotational correlation time of the triplet 
This article may be downloaded for personal use only. Any other use requires prior permission of the author and AIP Publishing. This article appeared in J. Chem. Phys. 152, 034202 (2020) and may be found at https://doi.org/10.1063/1.5133408

and $\omega_{0}$ the electron Larmor frequency. The latter approach was used here, with all parameters taken from ref 18, giving $P_{\mathrm{n}}=-108 P_{\text {eq. }}$. An additional hyperfine dependent polarization term $P_{\mathrm{m}}$ may be included in equation 20 to account for generation of multiplet polarization, ${ }^{38}$ but it has been experimentally demonstrated that only net polarization is significant for the xanthene - nitroxide systems discussed here. ${ }^{18}$

The expectation values of the electron and nuclear magnetization in the 4-level system take their familiar form representing the population differences,

$$
\begin{aligned}
& \left\langle S_{\mathrm{z}}\right\rangle=\left(N_{\beta \alpha}-N_{\alpha \alpha}\right)+\left(N_{\beta \beta}-N_{\alpha \beta}\right) \\
& \left\langle I_{\mathrm{z}}\right\rangle=\left(N_{\beta \alpha}-N_{\beta \beta}\right)+\left(N_{\alpha \alpha}-N_{\alpha \beta}\right)
\end{aligned}
$$

with their thermal equilibrium values calculated from the Boltzmann distribution for this system:

$$
\begin{gathered}
S_{\mathrm{z}}^{0}=\frac{\left(1+\exp \left(-\frac{\omega_{\mathrm{e}} \hbar}{k_{\mathrm{B}} T}\right)-\exp \left(-\frac{\omega_{\mathrm{n}} \hbar}{k_{\mathrm{B}} T}\right)-\exp \left(-\frac{\left(\omega_{\mathrm{e}}+\omega_{\mathrm{n}}\right) \hbar}{k_{\mathrm{B}} T}\right)\right.}{\left(1+\exp \left(-\frac{\omega_{\mathrm{e}} \hbar}{k_{\mathrm{B}} T}\right)+\exp \left(-\frac{\omega_{\mathrm{n}} \hbar}{k_{\mathrm{B}} T}\right)+\exp \left(-\frac{\left(\omega_{\mathrm{e}}+\omega_{\mathrm{n}}\right) \hbar}{k_{\mathrm{B}} T}\right)\right)} \\
I_{\mathrm{z}}^{0}=\frac{\left(1+\exp \left(-\frac{\omega_{\mathrm{n}} \hbar}{k_{\mathrm{B}} T}\right)-\exp \left(-\frac{\omega_{\mathrm{e}} \hbar}{k_{\mathrm{B}} T}\right)-\exp \left(-\frac{\left(\omega_{\mathrm{e}}+\omega_{\mathrm{n}}\right) \hbar}{k_{\mathrm{B}} T}\right)\right.}{\left(1+\exp \left(-\frac{\omega_{\mathrm{e}} \hbar}{k_{\mathrm{B}} T}\right)+\exp \left(-\frac{\omega_{\mathrm{n}} \hbar}{k_{\mathrm{B}} T}\right)+\exp \left(-\frac{\left(\omega_{\mathrm{e}}+\omega_{\mathrm{n}}\right) \hbar}{k_{\mathrm{B}} T}\right)\right)}
\end{gathered}
$$

At a magnetic field of $0.342 \mathrm{~T}$ and a temperature of $298.15 \mathrm{~K}$, these are $0.073046 \%$, and $0.000117 \%$, respectively.

The transition probabilities were calculated using the force-free hard sphere (FFHS) model. This model, first introduced by Hwang and Freed, considers the electron-nucleus interaction to occur solely by free diffusion, and is known to work well for DNP of protons. ${ }^{16,39}$ The transition probabilities are calculated as:

$$
\begin{aligned}
& w_{0}=k J\left(\omega_{\mathrm{I}}-\omega_{\mathrm{S}}, \tau_{\mathrm{c}}\right) \\
& w_{\mathrm{n}}=\frac{3}{2} k J\left(\omega_{\mathrm{I}}, \tau_{\mathrm{c}}\right) \\
& w_{2}=6 k J\left(\omega_{\mathrm{I}}+\omega_{\mathrm{S}}, \tau_{\mathrm{c}}\right)
\end{aligned}
$$

where $\tau_{\mathrm{c}}$ is the tumbling correlation time. In the FFHS model, this is redefined as the diffusion correlation time:

$$
\tau_{\mathrm{D}}=\frac{d^{2}}{D_{\mathrm{M}}+D_{\mathrm{L}}}
$$

where $D_{\mathrm{M}}$ and $D_{\mathrm{L}}$ are the correlation times of the translational diffusion coefficients of the radical, and the molecule bearing the nucleus to be polarized, respectively, and $d$ is the distance of closest approach between the nucleus and the unpaired electron. Literature values at room temperature for the related TEMPOL radical 
This article may be downloaded for personal use only. Any other use requires prior permission of the author and AIP Publishing. This article appeared in J. Chem. Phys. 152, 034202 (2020) and may be found at https://doi.org/10.1063/1.5133408

and water of $4.1 \times 10^{-10} \mathrm{~m}^{2} \mathrm{~s}^{-1}, 2.2995 \times 10^{-10} \mathrm{~m}^{2} \mathrm{~s}^{-1}$, and $d=2.5 \AA$, were used. ${ }^{8}$ The spectral densities were calculated according to:

$$
J\left(\omega, \tau_{\mathrm{D}}\right)=\frac{1+\frac{5 z}{8}+\frac{z^{2}}{8}}{1+z+\frac{z^{2}}{2}+\frac{z^{3}}{6}+\frac{4 z^{4}}{81}+\frac{z^{5}}{81}+\frac{z^{6}}{648}}
$$

where

$$
z=\sqrt{2 \omega \tau_{\mathrm{D}}}
$$

and the constant $k$ is

$$
k=\frac{32000 \pi}{405}\left(\frac{\mu_{0}}{4 \pi}\right)^{2} \frac{N_{A}[\mathrm{R}] \gamma_{I}^{2} \gamma_{S}^{2} S(S+1)}{d\left(D_{M}+D_{L}\right)}
$$

There has been significant debate over the appropriate form of the spectral density function in the case of Overhauser DNP due to discrepancy between values of the coupling factor determined by DNP and nuclear magnetic relaxation dispersion, ${ }^{24,40}$ but for the case of exclusively dipolar coupling and electronic relaxation times long compared to $\tau_{\mathrm{D}}$, as is the case for a nitroxide radical in water, it is appropriate to use the single spectral density function given above. ${ }^{40}$ Our calculations result in result in a coupling factor of $\xi=$ $\left(w_{2}-w_{0}\right) /\left(w_{0}+2 w_{\mathrm{n}}+w_{2}\right)=0.3674$ at $0.342 \mathrm{~T}$, which is consistent with the experimentally determined value for TEMPOL of $0.36,{ }^{24}$ justifying the use of the FFHS model with this parameter set. The simulation routine may be extended to account for variation in sample viscosity or magnetic field strength through recalculation of the transition probabilities, diamagnetic relaxation rate $1 / T_{1 \mathrm{n}}^{0}$ and $P_{\mathrm{n}}$, subject to use of an appropriate form of the spectral density function and knowledge of appropriate translational diffusion coefficients. Bearing in mind that the magnetic field dependence predicted by equation 22 has not to our knowledge been experimentally verified, and the necessity to correctly account for the field dependence of the relaxation rates, this worthwhile undertaking is beyond the scope of this initial study.

All simulations were carried out by numerical propagation of the coupled differential equations in $M_{a t l a b}{ }^{\circledR}$ 2017. Initially, an integration time step of $100 \mathrm{ps}$ was used, since this was found to give the optimum balance between accuracy and efficiency. Because both the triplet concentration and the electron magnetization reach a steady-state after approximately $10 \mu$ s, the only parameter that varies for most of the simulation is the nuclear magnetization, which varies much more slowly. Therefore, when the electron magnetization stopped changing by more than $0.001 \%$ during a single step, a longer time step of 100 ns was used to reduce computational load.

\section{Sample flow}

In the previously reported experiments the sample was flowed vertically upwards through the sample cell at $10 \mu \mathrm{l} \mathrm{min}{ }^{-1}$ to minimize the effects of photodegradation of the dye. ${ }^{17}$ However, this reduces the time 
period over which the sample is illuminated, as some sample will leave the illumination region during the experiment, and fresh sample will enter. To take these effects into account, we first ran the simulation for $20 \mathrm{~s}$ and stored the enhancement build-up curve. Then, the illumination region was divided into 1000 slices, with $u$ being the total volume below each slice. Thus, the exposure time for each slice, assuming a sufficiently long illumination period, is:

$$
t_{\mathrm{ex}}=\frac{u}{F}
$$

where $\mathrm{F}$ is the flow rate. If $t_{\mathrm{ex}}$ is greater than the experiment duration, then that maximum value is taken as the exposure time for that slice. By cross-referencing $t_{\mathrm{ex}}$ to the initial DNP build-up curve the average enhancement over the total sample volume was determined, reducing an initially observed over estimation of enhancements in the simulations compared to the experimental data.

\section{Pulsed illumination}

DNP using a pulsed laser was modelled as a repeated train of laser flashes, of length $\tau_{\mathrm{p}}$, followed by delays, where the laser was switched off, of length $\tau_{\mathrm{d}}$. The rate of triplet generation during each pulse was

$$
\begin{gathered}
\frac{d[\mathrm{~T}]}{d t}=P \frac{1}{C} \frac{\lambda}{h c} \frac{1}{V N_{\mathrm{A}}} \Phi\left(1-10^{-\epsilon[\mathrm{S}] l}\right)-k_{\mathrm{q}}[\mathrm{R}][\mathrm{T}]-k_{\mathrm{t}}[\mathrm{T}]-(2-\Phi) k_{\mathrm{tt}}[\mathrm{T}]^{2}-k_{\text {redox* }}[\mathrm{T}][\mathrm{S}] \\
-2 k_{\text {redox** }}[\mathrm{T}]^{2}-k_{\mathrm{sq}}[\mathrm{T}][\mathrm{S}]
\end{gathered}
$$

in which we have introduced a factor of $1 / C$, where $C$ is the duty cycle $\left(C=\tau_{\mathrm{p}} /\left(\tau_{\mathrm{p}}+\tau_{\mathrm{d}}\right)\right)$. This enables us to consider the case in which the time-averaged power is equal to that of a $\mathrm{CW}$ laser. Between pulses, the same equations are propagated, albeit without the triplet state build-up term.

Unlike in the continuous illumination case, the triplet concentration and electron magnetization do not reach a true steady state under pulsed illumination, so sufficient convergence to enable switching to a longer time step is never achieved. As direct propagation with high time resolution for the timescale of the nuclear polarization build-up is computationally expensive alternative approaches were used to increase efficiency. If the difference between the maximum saturation factors for each cycle and the previous remained below $10^{-10}$ for 10 consecutive cycles, the saturation factor was considered to have reached a pseudo-steady state. It was therefore not necessary to continue to propagate the triplet concentration and electron magnetization, as they were assumed to be identical for all subsequent cycles. The nuclear magnetization was then further propagated by repeatedly using the time profile of the electron magnetization for the last cycle. For simulations with very low duty cycles, the electron spin magnetization was found to relax to equilibrium between pulses. In these cases, to improve computational efficiency, the saturation factor was assumed to be zero for the remainder of the cycle after the difference between the magnetization and its equilibrium value came to be less than $10^{-7} \%$, allowing coarser time stepping until the next laser pulse.

\section{RESULTS AND DISCUSSION}


This article may be downloaded for personal use only. Any other use requires prior permission of the author and AIP Publishing. This article appeared in J. Chem. Phys. 152, 034202 (2020) and may be found at https://doi.org/10.1063/1.5133408

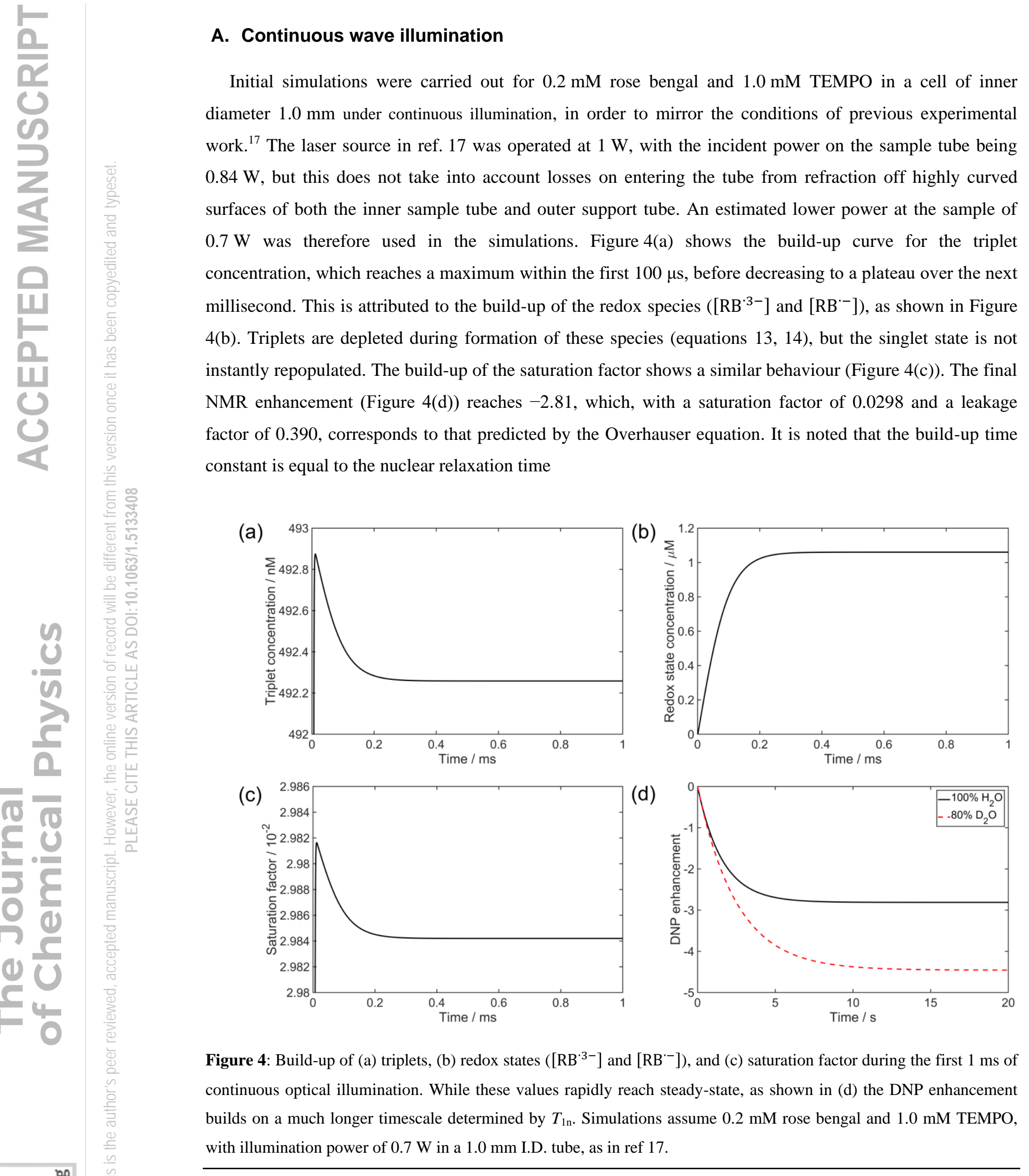



article appeared in J. Chem. Phys. 152, 034202 (2020) and may be found at https://doi.org/10.1063/1.5133408

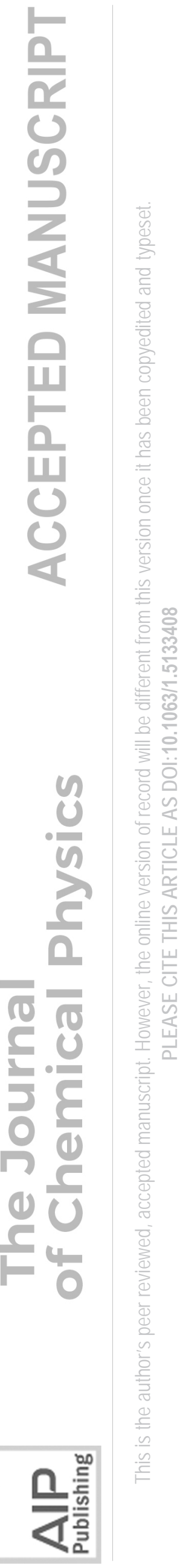

$(1.385 \mathrm{~s})$ as expected. Simulations were also carried out for a sample containing $80 \% \mathrm{D}_{2} \mathrm{O}$, where a $T_{1 \mathrm{n}}^{0}$ value of $6.542 \mathrm{~s}$ was assumed, leading to a leakage factor of 0.617 , which with unchanged saturation factor gives an NMR enhancement of -4.46 .

The dependence of the enhancement on the radical concentration, after illuminating for $10 \mathrm{~s}$ is shown in Figure 1(b). The magnitude of the enhancement values are close to the experimental values, and reproduce the finding of an optimum radical concentration. In the simulations for $100 \% \mathrm{H}_{2} \mathrm{O}$ solvent however the optimal concentration is $0.53 \mathrm{mM}$, slightly lower than the experimental value of $1.0 \mathrm{mM}$. In order to determine why this is the case, we examined the leakage and saturation factors. Figure 1(c) shows that the leakage factor increases with increasing radical concentration, as is expected. The experimental values are reasonably reproduced, although slightly outside of experimental uncertainty range. This is likely to be due to errors in the experimental measurement of the relaxation times performed in a low-field EPR electromagnet of lower stability and homogeneity than a conventional NMR magnet, ${ }^{17}$ and being consistent across the concentration range should not affect the conditions for maximum enhancement. The source of the discrepancy therefore seems to be the calculation of the saturation factors. Figure 1(d) shows that the saturation factor decreases with increasing radical concentration; this is because the increased intermolecular quenching at higher radical concentrations reduce the steady-state triplet concentration. It is this compromise between increased leakage factor and reduced saturation factor that determines the optimum radical concentration. It is notable that while the simulations reproduce the experimental saturation factor at high radical concentrations, the simulated values are significantly higher at low radical concentrations. The source of this discrepancy is postulated to be experimental, arising from heating effects, or photodegradation of the dye. Inclusion of an additional loss term is able to improve agreement but given the nature of the postulated degradation process is entirely unknown we did not consider use of this phenomenological term justified. It should also be noted that the experimental saturation factors were not measured directly, but calculated from the measured signal enhancement and leakage parameter, increasing uncertainty in the experimental values. Nevertheless, it is promising that the simulations are able to reproduce the magnitudes of the enhancements without need for any free fitting parameters.

To aid in rationalization and optimization of enhancements through the optical DNP approach it is convenient to derive an analytical expression for the saturation factor as is possible for the microwave-driven approach. The steady-state solution of equation 20 may be written as:

$$
s_{\mathrm{ss}}=-\frac{P_{\mathrm{n}}}{P_{\mathrm{eq}}} k_{\mathrm{q}} T_{1 \mathrm{e}}[\mathrm{T}]_{\mathrm{ss}}
$$

noting that $S_{\mathrm{z}}^{0}=P_{\mathrm{eq}}[R]$. To obtain the steady-state triplet concentration $[\mathrm{T}]_{\mathrm{ss}}$, we make the approximation that ground-state depletion is negligible, and thus:

$$
[\mathrm{T}]_{\mathrm{ss}}=\frac{2 X}{A+\sqrt{A^{2}+4 B X}}
$$


where

$$
\begin{aligned}
& A=k_{\mathrm{q}}[\mathrm{R}]+k_{\mathrm{t}}+k_{\text {redox* }}[\mathrm{S}]+k_{\mathrm{sq}}[\mathrm{S}] \\
& B=(2-\Phi) k_{\mathrm{tt}}+2 k_{\text {redox } * *} \\
& X=P \frac{\lambda}{h c} \frac{1}{V N_{\mathrm{A}}} \Phi\left(1-10^{-\epsilon[\mathrm{S}] l}\right)
\end{aligned}
$$

Under conditions mirroring the previous experimental study, with a TEMPO concentration of $1.0 \mathrm{mM}$, the steady state saturation factor is calculated to be 0.0297 , in good agreement with that from a numerical propagation of the system as described above. While equation 36 should be applicable to any dye, equation 37 is based on knowledge of the photochemistry of rose bengal, though similar expressions could be derived for other systems subject to requisite knowledge of the photochemical pathways available.

In order to determine a route to maximisation of the optical DNP enhancement it is instructive to consider the separate components of equation 37 . The radical concentration $[R]$ appears in the $A$ term (equation 37b) which is seen to make both positive and negative contributions to the steady-state triplet yield. Simple manipulation of this concentration does not therefore provide a route to achieve an increased enhancement factor, as already discussed above. The $B$ term contains only photochemical constants relating to the nature of the triplet and will not be considered further, whereas the $X$ term relates to triplet generation and the effect of laser power. With the simplifying assumption that the sample is not flowed we consider the effect on the saturation and enhancement factors of doubling the laser power at the sample, Figure 5. This suggests a significant increase in the achieved enhancement, though it is important to consider the excessive power may cause photodegradation and significant sample heating, as has also plagued the microwave DNP approach.

(a)

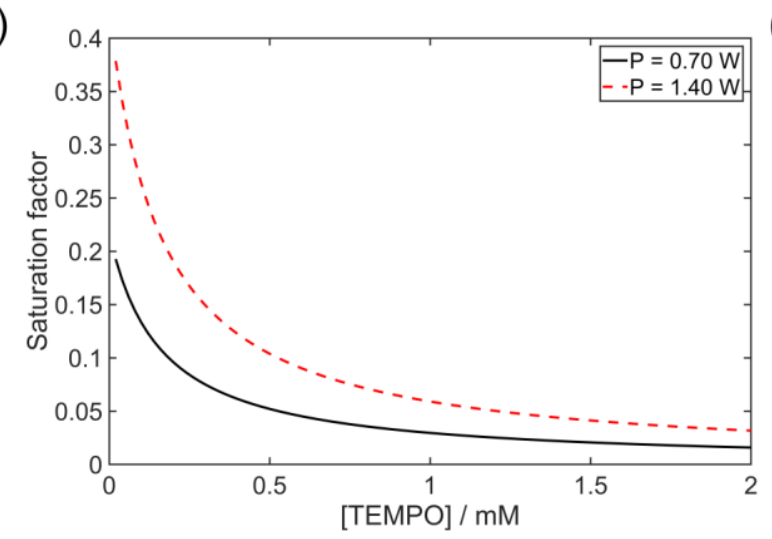

(b)

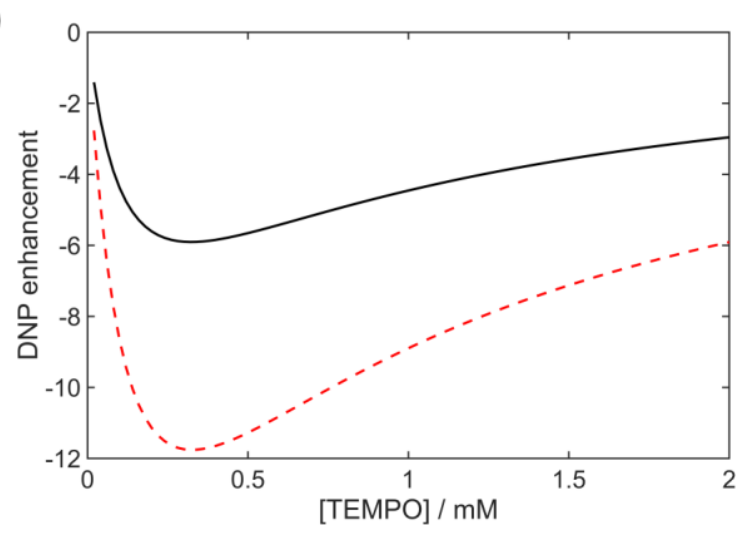

Figure 5: Simulated (a) saturation factor and (b) DNP enhancement for laser powers of $0.70 \mathrm{~W}$ and $1.40 \mathrm{~W}$, with a constant rose bengal concentration of $0.2 \mathrm{mM}$ in an $80 \% \mathrm{D}_{2} \mathrm{O}$ solution. 
This article may be downloaded for personal use only. Any other use requires prior permission of the author and AIP Publishing. This article appeared in J. Chem. Phys. 152, 034202 (2020) and may be found at https://doi.org/10.1063/1.5133408

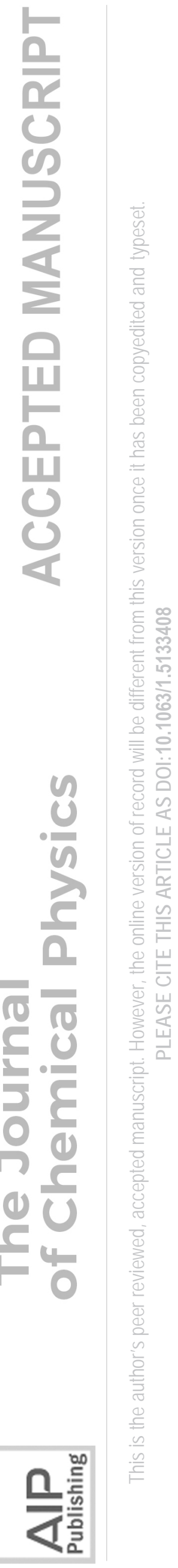

(a)

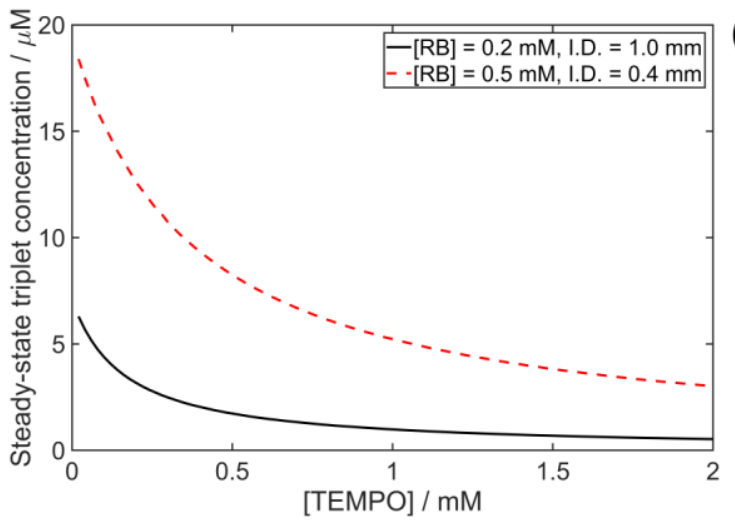

(b)

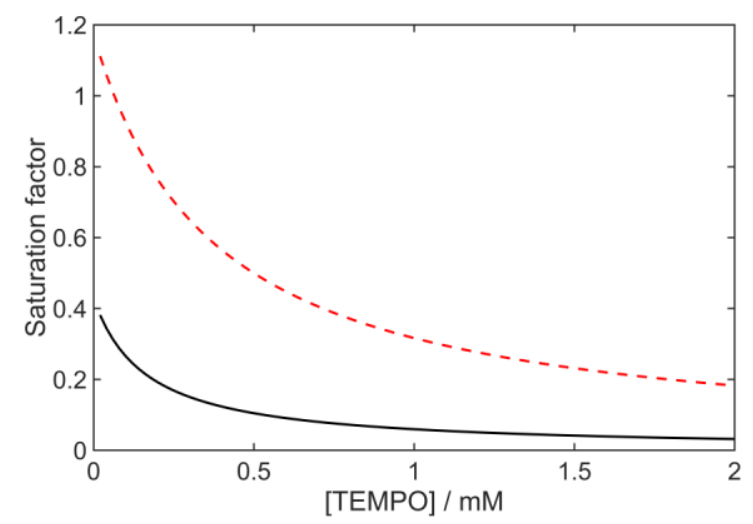

(c)

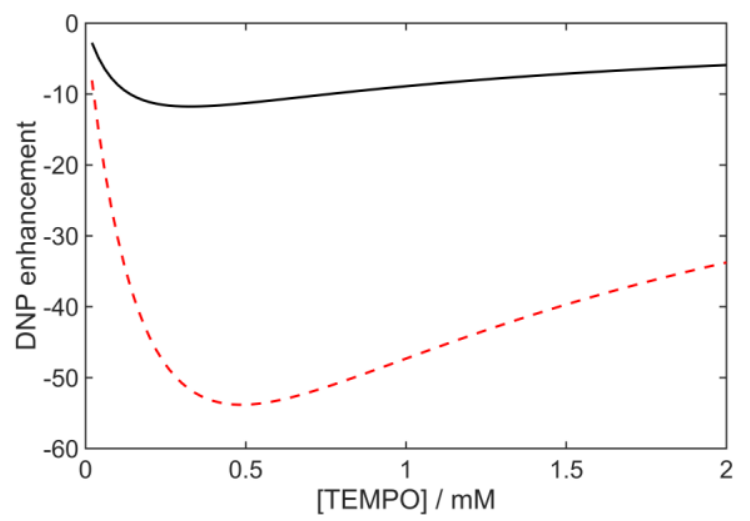

Figure 6: Simulated (a) triplet concentration, (b) saturation factor and (c) DNP enhancement for $0.2 \mathrm{mM}$ rose bengal in a $1.0 \mathrm{~mm}$ I.D. tube, and $0.5 \mathrm{mM}$ rose bengal in a $0.4 \mathrm{~mm}$ I.D. tube, both of which provide an absorbance of 0.75 at the illumination wavelength. The laser power was $1.40 \mathrm{~W}$, and the solution contained $80 \% \mathrm{D}_{2} \mathrm{O}$.

The triplet generation term may also be increased through use of higher dye concentrations to increase the optical absorbance. It has however already been shown experimentally that increasing the concentration too much actually decreases the enhancement, since the ability of light to penetrate the sample is reduced. ${ }^{17}$ Due to the complexity of modelling penetration effects these were not explicitly included in the numerical simulation; instead we assume the sample to be homogeneous but require that the optical absorbance should not exceed unity. Considering the average over all path lengths in cylindrical tubes it was determined that the average absorbance of 0.75 for $0.2 \mathrm{mM}$ rose bengal in a $1.0 \mathrm{~mm}$ I.D. tube (average path length $0.78 \mathrm{~mm}$ ) is identical to that for $0.5 \mathrm{mM}$ rose bengal if the tube diameter is reduced to $0.4 \mathrm{~mm}$ (average path length $0.31 \mathrm{~mm}$ ). Simulations under these conditions are shown in Figure 6, from which it may be seen that despite the constant absorbance the latter sample has an almost five-fold higher enhancement. This effect is attributed to the fact that in reducing the path length to obtain constant absorbance there is an approximately six-fold reduction in the absorbance volume. As seen in equation 37c the $X$ term is inversely proportional to $V$, therefore there is an overall increase in the concentration of triplets (Figure 6a) due to the higher power density. While this is partially offset by increased self-quenching the higher triplet concentration shifts the delicate balance of competing pathways to favour a moderate increase in the optimal radical concentration, and overall leads to considerable improvement in the achieved enhancement. This prediction warrants future experimental investigation, ideally in combination with microfluidic NMR probe designs to avoid 
This article may be downloaded for personal use only. Any other use requires prior permission of the author and AIP Publishing. This article appeared in J. Chem. Phys. 152, 034202 (2020) and may be found at https://doi.org/10.1063/1.5133408

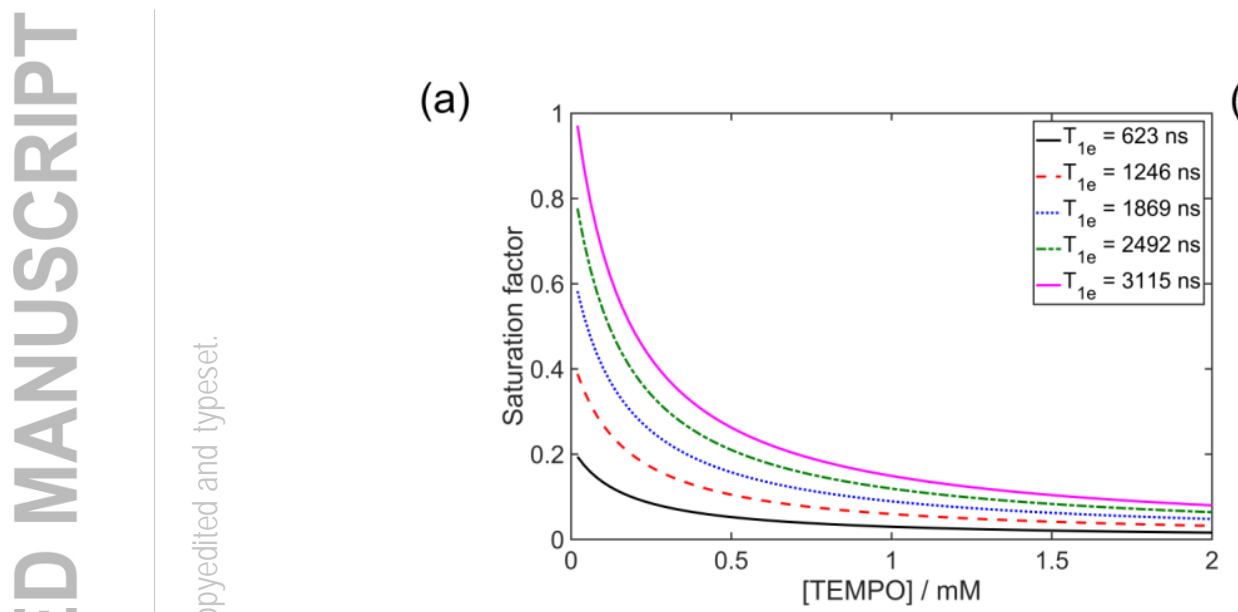

(b)

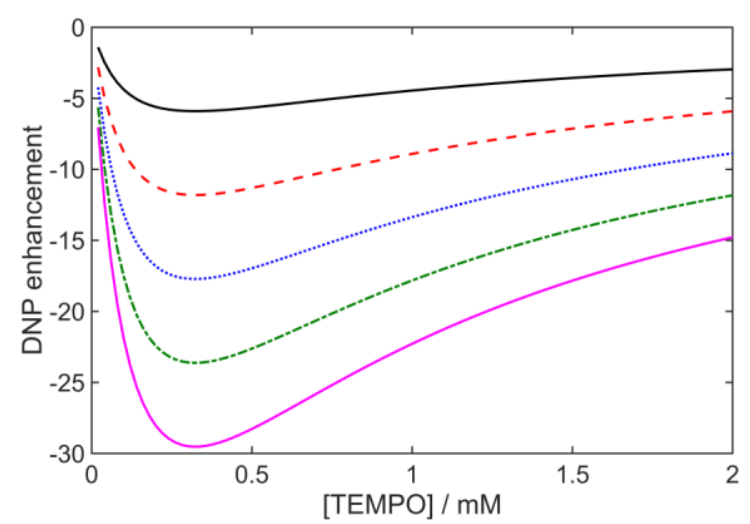

Figure 7: (a) Saturation factor and (b) DNP enhancement for various electron spin-lattice relaxation times, chosen as multiples of the experimentally determined value for the TEMPO radical. The rose bengal concentration was kept constant at $0.2 \mathrm{mM}$, in a 20:80 $\mathrm{H}_{2} \mathrm{O}: \mathrm{D}_{2} \mathrm{O}$ solution in a $1.0 \mathrm{~mm}$ I.D. tube, with a laser power of $0.7 \mathrm{~W}$.

compromise in filling factor and hence detection sensitivity, and is potentially a result of great importance given investigation of volume limited samples is a key application area for DNP enhanced NMR.

Another factor strongly influencing the enhancement is the electronic relaxation time. As shown in equation 36, and confirmed in Figure 7, there is a linear relationship between $T_{1 \mathrm{e}}$ and the saturation factor. Manipulation of relaxation rates may be achieved by changing experimental conditions such as solvent viscosity and magnetic field but the most significant improvements are likely to be seen through choice of a more slowly relaxing radical. In all cases there are likely to also be effects on $T_{1 \mathrm{n}}$ and $P_{\mathrm{n}}$, but if a more slowly relaxing radical can be used without compromising the RTPM efficiency, perhaps through simple isotopic substitution of the nitroxide, ${ }^{41}$ this ought to significantly increase the enhancement achieved. The search for more slowly relaxing radicals that generate significant net polarization via the RTPM, while not compromising the DNP saturation factor, must be a key target for future development of the optical Overhauser DNP method.

\section{B. Pulsed illumination}

While experimental demonstration of optical DNP has so far been confined to continuous illumination it is interesting to consider whether there are benefits from pulsed illumination as have been described in the microwave pumping mode. ${ }^{21}$ For example laser heating effects could potentially be minimised if continuous illumination can be replaced by low duty cycle pulses in order to allow cooling delays. Figure 8 shows the 
This article may be downloaded for personal use only. Any other use requires prior permission of the author and AIP Publishing. This article appeared in J. Chem. Phys. 152, 034202 (2020) and may be found at https://doi.org/10.1063/1.5133408

(a)

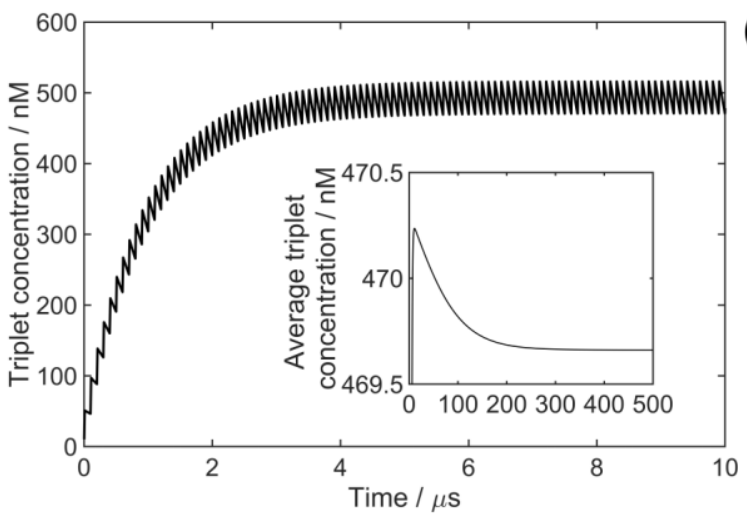

(b)

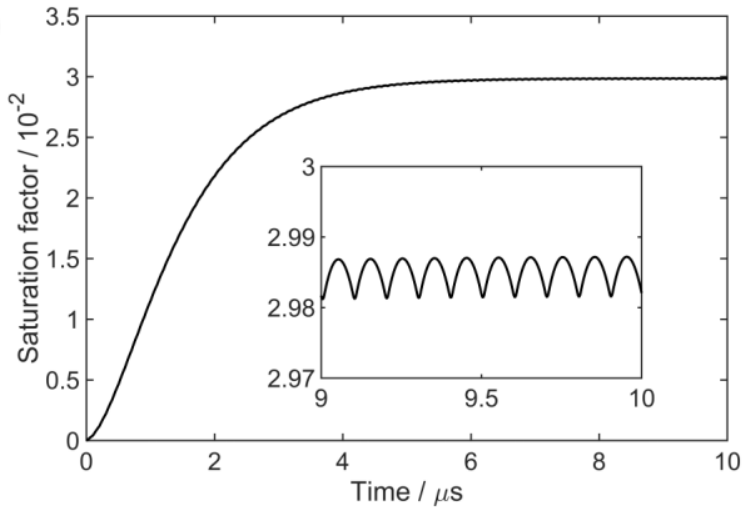

(c)

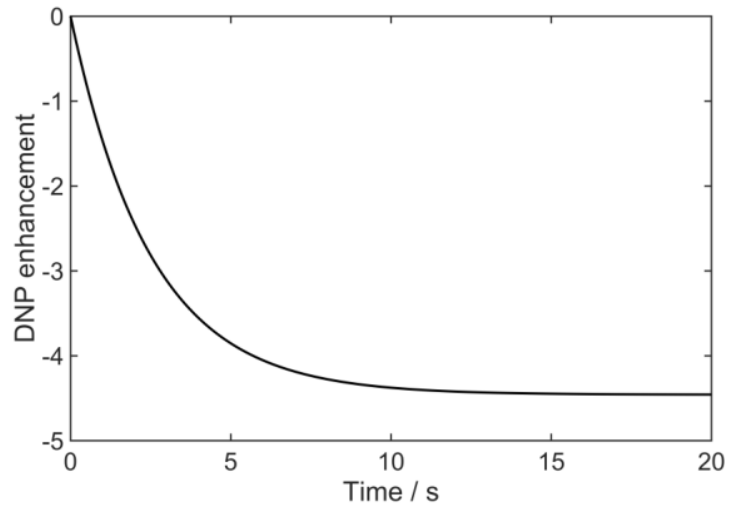

Figure 8: Pulsed optical DNP simulation showing build-up of (a) triplet yield (b) saturation factor and (c) DNP enhancement. The inset in (a) shows the marginal overshoot in the initial build-up of the average triplet concentration above the eventual pseudo steady-state value while the inset in (b) magnifies the continued small oscillations about the pseudo steady-state value. Simulation parameters are pulse length $10 \mathrm{~ns}, 10 \%$ duty cycle and average illumination power of $0.7 \mathrm{~W}$. $1.0 \mathrm{mM}$ TEMPO. $0.2 \mathrm{mM}$ rose bengal in $80 \% \mathrm{D}_{2} \mathrm{O}$ solution in $1.0 \mathrm{~mm}$ I.D. tube.

build-up curves for the triplet yield, saturation factor, and DNP enhancement calculated for a laser pulse length of $10 \mathrm{~ns}$, and a $10 \%$ duty cycle with average power of $0.7 \mathrm{~W}$. Other experimental parameters are identical to those optimised in ref. 17 and used above, namely $1.0 \mathrm{mM}$ TEMPO and $0.2 \mathrm{mM}$ rose bengal in a $80 \% \mathrm{D}_{2} \mathrm{O}$ solution, considering a sample tube of $1.0 \mathrm{~mm}$ inner diameter. As expected, the triplet concentration (Figure 8(a)) oscillates, due to triplet generation during illumination pulses, and decay during delay (dark) periods. The saturation factor also oscillates (Figure 8(b), inset), although the oscillations are proportionally smaller and dampened since triplets are still present, and hence DEP is generated through triplet quenching, during the illumination delays. As with the CW simulations (Figure 4), the saturation factor and triplet concentration reach a maximum within a few microseconds, before falling marginally to achieve a pseudo-steady-state value over the course of a few hundred microseconds, as shown for the triplet in the inset to Figure 8(a). Damped oscillations are also seen for the enhancement factor, which reaches a pseudo-steady-state much more slowly with a time constant equal to the nuclear relaxation time $T_{\mathrm{ln}}$, and the overall enhancement achieved is comparable to the $\mathrm{CW}$ case. 
This article may be downloaded for personal use only. Any other use requires prior permission of the author and AIP Publishing. This article appeared in J. Chem. Phys. 152, 034202 (2020) and may be found at https://doi.org/10.1063/1.5133408

Because the NMR timescale is much longer than the EPR timescale, the overall DNP enhancement factor depends on the average saturation factor, and by extension triplet yield, over one illumination cycle. We consider how these are affected by the illumination pulse length and duty cycle, beginning with comparison to the effects reported for pulsed microwave DNP by Vieth and co-workers. ${ }^{20,21}$ They derived an expression for the overall enhancement under pulsed microwave irradiation:

$$
\varepsilon=\frac{\xi f s_{\max } T_{1 \mathrm{e}}}{\tau} \times \frac{(1-\cos \varphi)\left(1-\exp \left(-2 \tau_{\mathrm{d}} / T_{1 \mathrm{e}}\right)\right)}{1-2 \exp \left(-\tau_{\mathrm{d}} / T_{1 \mathrm{e}}\right) \cos \varphi+\exp \left(-2 \tau_{\mathrm{d}} / T_{1 \mathrm{e}}\right)} \times \frac{\gamma_{\mathrm{e}}}{\gamma_{\mathrm{n}}}
$$

where $\varphi$ is the flip angle, $\varphi=\gamma_{\mathrm{e}} B_{1} \tau_{\mathrm{p}}$, with $\tau_{\mathrm{p}}$ and $\tau_{\mathrm{d}}$ pulse and delay length respectively, $B_{1}$ the microwave field strength and $s_{\max }$ is a maximum saturation factor dependent on the number of hyperfine lines subject to on-resonance irradiation. This results in two components to the pulse length dependence of the enhancement: Firstly, with variation in microwave pulse length (Figure 9(a)) an oscillatory behaviour is seen, with saturation maximum when $\varphi=\pi, 2 \pi, 5 \pi, \ldots$, and zero when $\varphi=0,2 \pi, 4 \pi, \ldots$ Secondly, with a fixed pulse length the enhancement decreases as the delay becomes longer (lower duty cycle), due to relaxation effects. In contrast, in optically pumped DNP the EPR transitions are not irradiated directly, so coherent manipulation of the electron spin system is not achieved, hence no oscillatory dependence of the average triplet concentration, saturation factor or enhancement on the illumination pulse length is observed (Figure 9(b)-(d)). The assumption that no electron spin polarization is generated during the illumination delays is also no longer valid, since triplets will still be present during this period. The dependence on both illumination pulse length and duty cycle is therefore rather small $(<2 \%)$ over the same duty cycle range and with five orders of magnitude variation in pulse length, under the condition of constant average illumination power.

Given the high computational expense of propagating the full electron-nuclear spin-system, to further investigate pulse length effects in optical DNP we derive a simple analytical expression for the average enhancement under pseudo steady-state conditions:

$$
\langle\varepsilon\rangle=-\xi f\langle s\rangle\left|\frac{\gamma_{\mathrm{e}}}{\gamma_{\mathrm{n}}}\right|
$$

where

$$
\langle s\rangle=-\frac{P_{\mathrm{n}}}{P_{\mathrm{eq}}} k_{\mathrm{q}}\langle[\mathrm{T}]\rangle T_{1 \mathrm{e}} .
$$

As in the $\mathrm{CW}$ case, it is assumed that ground-state depletion is negligible and, in addition, since the total triplet concentration is small we discount terms that are second order with respect to the triplet concentration $\left(k_{\mathrm{tt}}\right.$ and $\left.k_{\mathrm{redox}} * *\right)$. After convergence, the average triplet concentrations over the pulse and the delay are:

$$
\langle[\mathrm{T}]\rangle_{\mathrm{p}}=\frac{X^{\prime} A \tau_{\mathrm{p}}-[\mathrm{T}]_{\mathrm{st}} A \exp \left(-A \tau_{\mathrm{p}}\right)+X^{\prime} \exp \left(-A \tau_{\mathrm{p}}\right)+[\mathrm{T}]_{\mathrm{st}} A-X^{\prime}}{Y^{2} \tau_{\mathrm{p}}}
$$




$$
\langle[\mathrm{T}]\rangle_{\mathrm{d}}=\frac{[\mathrm{T}]_{\mathrm{st}}{ }^{\prime}}{Y \tau_{\mathrm{d}}}\left(1-\exp \left(-A \tau_{\mathrm{d}}\right)\right)
$$

where

$$
X^{\prime}=\frac{P}{C} \frac{\lambda}{h c} \frac{1}{V N_{\mathrm{A}}} \Phi\left(1-10^{-\epsilon[\mathrm{S}] l}\right)
$$

and $A$ was previously defined by equation $37 \mathrm{~b}$. The average triplet yield over the entire pulse is the weighted average of these:

$$
\langle[\mathrm{T}]\rangle=\frac{P \lambda \Phi\left(1-10^{-\varepsilon[\mathrm{S}] L}\right)}{V N_{\mathrm{A}} h c\left(k_{\mathrm{q}}[\mathrm{R}]+k_{\mathrm{t}}+k_{\mathrm{sq}}[\mathrm{S}]+k_{\mathrm{redox} *}[\mathrm{~S}]\right)}
$$

and hence:

$$
\langle\varepsilon\rangle=\frac{P \lambda \Phi\left(1-10^{-\varepsilon[\mathrm{S}] L}\right)}{V N_{\mathrm{A}} h c\left(k_{\mathrm{q}}[\mathrm{R}]+k_{\mathrm{t}}+k_{\mathrm{sq}}[\mathrm{S}]+k_{\text {redox* }}[\mathrm{S}]\right)} \frac{P_{\mathrm{n}}}{P_{\mathrm{eq}}} k_{\mathrm{q}} T_{1 \mathrm{e}} \xi f\left|\frac{\gamma_{\mathrm{e}}}{\gamma_{\mathrm{n}}}\right|
$$

The intriguing result is an expression that does not depend on either the pulse length or the duty cycle, and in fact, gives an enhancement equal to the continuous wave value. As Figure 9(e) shows, if the assumptions we have made are taken into account in the numerical simulation, then this effect is reproduced, and while the assumptions introduce a small numerical error the average triplet concentration is indeed independent of pulse length in the limit of short pulses. This is a simple reflection of the condition of constant average illumination power, and hence constant time averaged photon flux through the sample. Deviations for longer pulse lengths and lower duty cycles are seen to arise as under these conditions the transient triplet concentration is able to periodically build to a level under which second order decay processes and ground state depletion become significant (Figure 9(f)). Nevertheless, equation 45 is a convenient approximation to determine the maximum expected magnitude of the optical DNP enhancement under pulsed illumination conditions based on known concentrations and kinetic parameters.

Initial attempts to observe optical DNP used high-power pulsed lasers common to TR-EPR experiments, to generate significant transient electron spin magnetization, yet optical DNP was not observed under these conditions. To explain this observation consider for example an Nd:YAG laser with $10 \mathrm{~ns}$ pulse width and $20 \mathrm{~Hz}$ repetition rate, which is typical of the type used for TR-EPR experiments - the duty cycle in this case is very low $\left(2 \times 10^{-5} \%\right)$, hence it is necessary to extend the range of our simulations to lower duty cycles, Figure 10. With a constant pulse length the triplet yield, saturation factor, and enhancement all decrease sharply as the duty cycle is decreased towards this low duty cycle regime. This may seem counterintuitive since with a constant time averaged laser power the number of triplets generated per pulse increases with a lower duty cycle (Figure 10(d)). At low duty cycles however the pulse delays become long compared to the triplet lifetime $\left(1 / k_{\mathrm{t}}\right)$, hence the time averaged triplet concentration tends to zero (Figure 10(a)). Unless the 
This article may be downloaded for personal use only. Any other use requires prior permission of the author and AIP Publishing. This article appeared in J. Chem. Phys. 152, 034202 (2020) and may be found at https://doi.org/10.1063/1.5133408
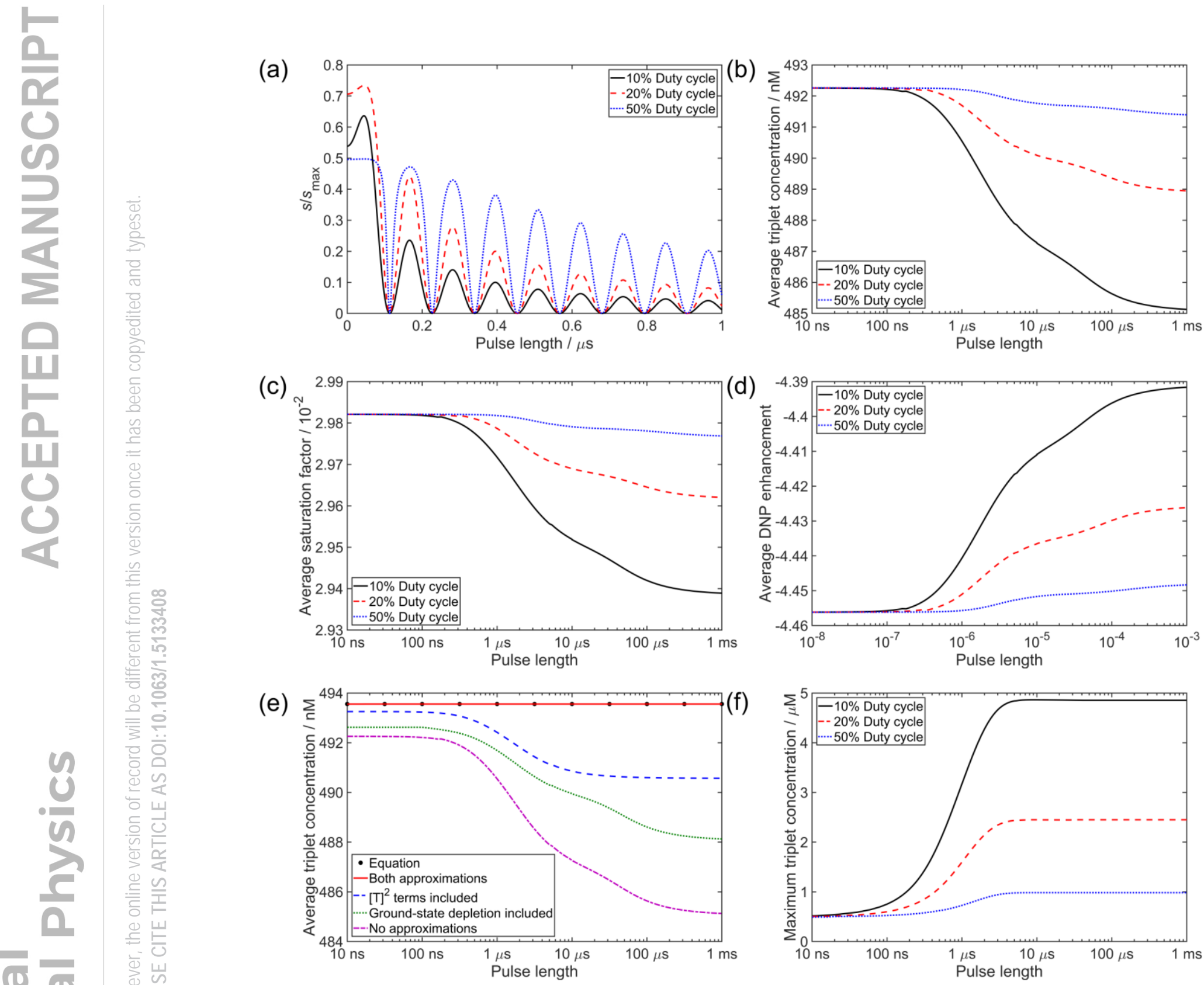

Figure 9: Dependence of (a) the normalised saturation factor on the pulse length in the microwave pumping case for a $B_{1}$ field of $0.1 \mathrm{mT}$, derived using equations from Ref. 32, and dependence of the average (b) triplet yield, (c) saturation factor, and (d) enhancement factor on pulse length for different illumination duty cycles as indicated. (e) shows the influence of various approximations used in the derivation of equation 45 when calculating the average triplet yield for a $10 \%$ duty cycle, and (f) the maximum triplet concentration per cycle under the same conditions as (b)-(d). For all optical DNP simulations the illumination power is held constant at $0.7 \mathrm{~W}$, with other simulation parameters as in Figure 8 .

electronic relaxation time is long compared to the pulse delay the electron spin system can then fully relax between pulses, with any portion of the delay period after full electronic relaxation contributing to decay of the nuclear polarization, reducing the maximum DNP enhancement achievable. What is interesting to note is that especially for short pulses there is a significant duty cycle range for which the achievable enhancement is approximately equal to the continuous illumination value. The use of high repetition pulsed laser sources 
This article may be downloaded for personal use only. Any other use requires prior permission of the author and AIP Publishing. This article appeared in J. Chem. Phys. 152, 034202 (2020) and may be found at https://doi.org/10.1063/1.5133408

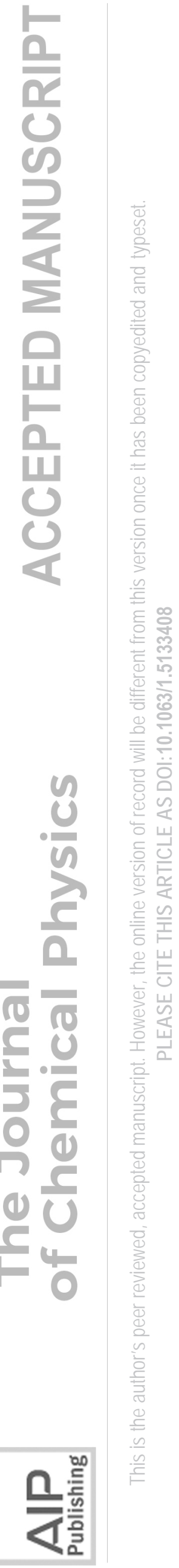

(a)

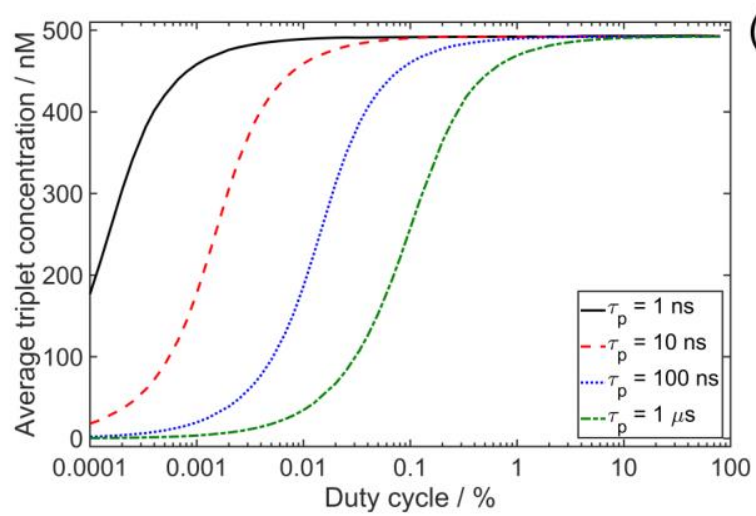

(c)

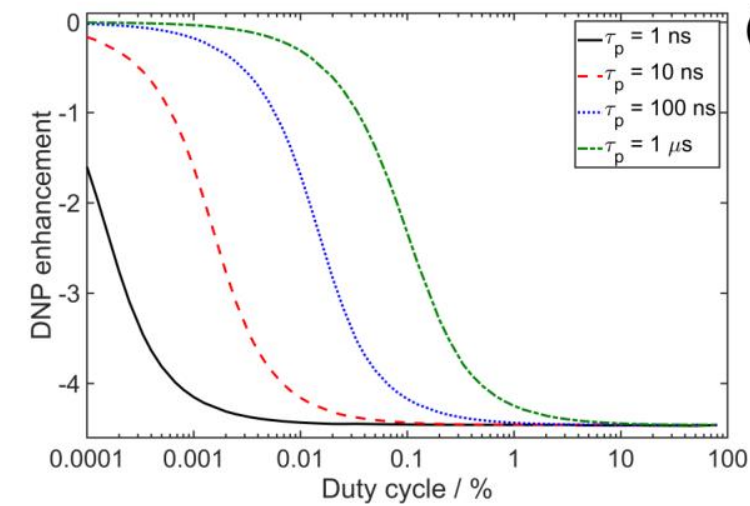

(b)

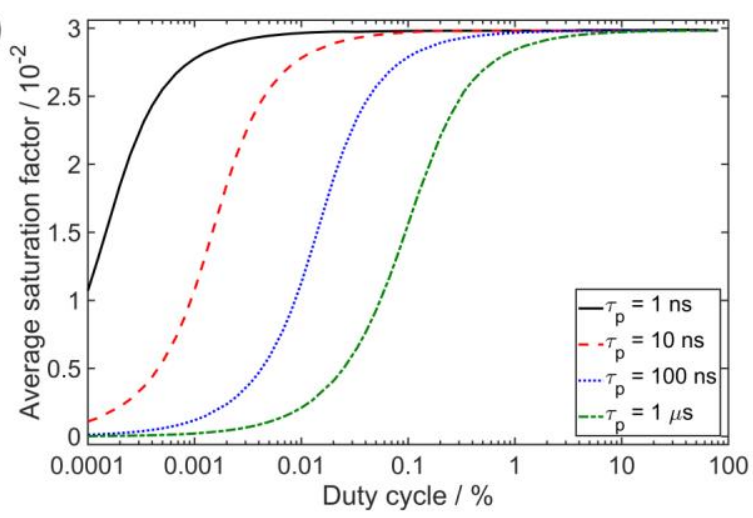

(d)

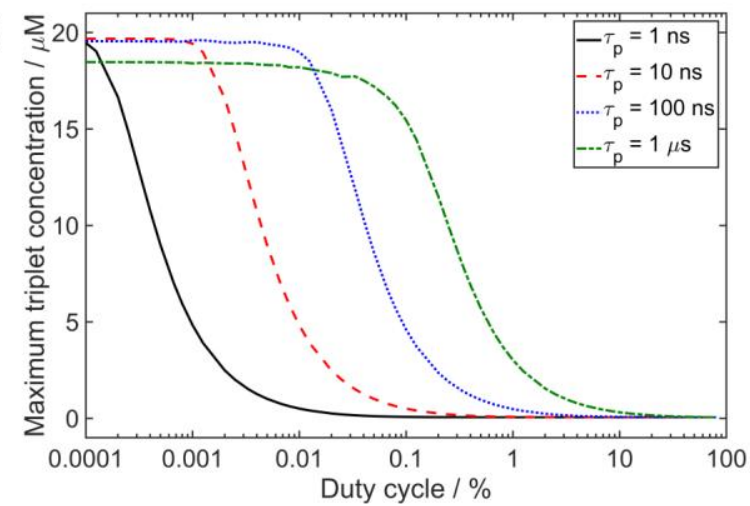

Figure 10: Dependence of the average (a) triplet yield, (b) saturation factor, (c) enhancement factor, and (d) maximum triplet yield on the illumination duty cycle for various pulse lengths as indicated.

may therefore be beneficial, permitting use of relatively low illumination duty cycles (e.g. $0.01 \%$ ) to reduce heating effects, without compromising the enhancement obtained. Use of more slowly relaxing radicals and triplets ought to extend the high enhancement window to lower duty cycles, and should be a target for future optimization of the radical-triplet system used in the optical DNP approach

\section{CONCLUSIONS}

In this work we have developed the first numerical simulations to describe Overhauser DNP in the solution state in the case of optical rather than microwave pumping. Combining Bloch and kinetic equations previously used to model the results of TR-EPR experiments with the Solomon equations describing electron-nuclear cross-relaxation, and solving numerically, the previously reported experimental results for optical Overhauser DNP have been reproduced from first principles. Through an initial analysis of the optical DNP effect as a function of experimental parameters such as radical and dye concentrations, laser power, and sample geometry, we have identified considerable scope for further increases in the accessible NMR enhancements. The most important finding in this regard is that by reducing sample volume and optical path length it may be possible to utilize higher dye concentrations, resulting in an increased concentration of photogenerated triplets and hence greater optical DNP enhancement. In combination with microfluidic NMR methods the optical Overhauser DNP method therefore provides potential for significant 
This article may be downloaded for personal use only. Any other use requires prior permission of the author and AIP Publishing. This article appeared in J. Chem. Phys. 152, 034202 (2020) and may be found at https://doi.org/10.1063/1.5133408

sensitivity enhancements for volume limited NMR samples that could be challenging to measure under standard NMR conditions.

We have also investigated the possibility of using pulsed illumination in optical Overhauser DNP. While coherence effects are absent from the optical approach, we found that the achievable enhancement is invariant to illumination pulse length and duty cycle over a wide range, provided pulse delays do not become long with respect to the triplet and electron spin polarization lifetimes. Within this regime time-averaged laser power was found to be the key determinant of the ultimate NMR enhancement, with negligible reduction in enhancement compared to the continuous wave regime. Extension of the experimental method from continuous wave to lower duty cycle pulsed illumination potentially reduces the potential for undesirable heating effects. Dye photobleaching is an additional potential challenge to the optically pumped DNP method which could be overcome by use of an appropriate system for dye regeneration, ${ }^{42}$ which may be a more effective if regeneration can occur during the delay periods of lower duty cycle pulsed illumination.

Finally, analytical expressions were provided for the enhancement achieved in both pulsed and continuous illumination experiments, making use of simplifying assumptions regarding ground state depletion and higher order decay processes. Given breakdown of these assumptions leads to overestimation of the triplet concentration these expressions provide a convenient and fast way of determining the maximum potential enhancement, and while derived in the case of the specific known photochemistry of the rose bengal system could be extended for other dye systems provided the dominant photochemical pathways are known. This will be a powerful tool for the screening of polarizing systems to determine their potential for application in the optical Overhauser DNP method. The simulation approach will also be applied to consider the magnetic field dependence of the combined polarization mechanisms so as to determine the optimal field for the application of optically generated DNP. This challenging undertaking will necessitate careful choice of appropriate forms of the spectral density functions and experimental verification of Shushin's equation for the magnitude of net RTPM polarization.

\section{ACKNOWLEDGEMENTS}

DJC thanks the School of Applied Sciences at the University of Huddersfield for a departmentally funded studentship.

\section{REFERENCES}

${ }^{1}$ J.H. Lee, Y. Okuno, and S. Cavagnero, J. Magn. Reson. 241, 18 (2014).

${ }^{2}$ A.W. Overhauser, Phys. Rev. 92, 411 (1953).

${ }^{3}$ T.R. Carver and C.P. Slichter, Phys. Rev. 92, 212 (1953).

${ }^{4}$ H.G. Beljers, L. van der Kint, and J.S. van Wieringen, Phys. Rev. 95, 221 (1954). 
This article may be downloaded for personal use only. Any other use requires prior permission of the author and AIP Publishing. This article appeared in J. Chem. Phys. 152, 034202 (2020) and may be found at https://doi.org/10.1063/1.5133408

5 T. Maly, G.T. Debelouchina, V.S. Bajaj, K.-N. Hu, C.-G. Joo, M.L. Mak-Jurkauskas, J.R. Sirigri, P.C.A. van der Wel, J. Herzfeld, R.J. Temkin, and R.G. Griffin, J. Chem. Phys. 128, 1 (2008).

${ }^{6}$ R.G. Griffin and T.F. Prisner, Phys. Chem. Chem. Phys. 12, 5737 (2010).

${ }^{7}$ J. Van Bentum, B. Van Meerten, M. Sharma, and A. Kentgens, J. Magn. Reson. 264, 59 (2016).

${ }^{8}$ P.J.M. van Bentum, G.H.A. van der Heijden, J.A. Villanueva-Garibay, and A.P.M. Kentgens, Phys. Chem. Chem. Phys. 13, 17831 (2011).

${ }^{9}$ P. Höfer, G. Parigi, C. Luchinat, P. Carl, G. Guthausen, M. Reese, T. Carlomagno, C. Griesinger, and M. Bennati, J. Am. Chem. Soc. 130, 3254 (2008).

${ }^{10}$ M.J. Prandolini, V.P. Denysenkov, M. Gafurov, B. Endeward, and T.F. Prisner, J. Am. Chem. Soc. 131, 6090 (2009).

${ }^{11}$ M.-T. Turke, I. Tkach, M. Reese, P. Höfer, and M. Bennati, Phys. Chem. Chem. Phys. 12, 5893 (2010).

12 J.A. Villanueva-Garibay, G. Annino, P.J.M. van Bentum, and A.P.M. Kentgens, Phys. Chem. Chem. Phys. 12, 5846 (2010).

${ }^{13}$ J.M. Franck, A. Pavlova, J.A. Scott, and S. Han, Prog. Nucl. Magn. Reson. Spectrosc. 74, 33 (2013).

${ }^{14}$ J.R. Biller, R. Barnes, and S. Han, Curr. Opin. Colloid Interface Sci. 33, 72 (2018).

15 J.G. Krummenacker, V.P. Denysenkov, and T.F. Prisner, Appl. Magn. Reson. 43, 139 (2012).

${ }^{16}$ E. Ravera, C. Luchinat, and G. Parigi, J. Magn. Reson. 264, 78 (2016).

${ }^{17}$ M.W. Dale and C.J. Wedge, Chem. Commun. 52, 13221 (2016).

${ }^{18}$ H. Takahashi, M. Iwama, N. Akai, K. Shibuya, and A. Kawai, Mol. Phys. 112, 1012 (2014).

${ }^{19}$ G. Liu, S.-H. Liou, N. Enkin, I. Tkach, and M. Bennati, Phys. Chem. Chem. Phys. 19, 31823 (2017).

${ }^{20}$ S.E. Korchak, A.S. Kiryutin, K.L. Ivanov, A. V Yurkovskaya, Y.A. Grishin, H. Zimmermann, and H.M. Vieth, Appl. Magn. Reson. 37, 515 (2010).

${ }^{21}$ E.A. Nasibulov, K.L. Ivanov, A. V. Yurkovskaya, and H.M. Vieth, Phys. Chem. Chem. Phys. 14, 6459 (2012).

${ }^{22}$ K.. Hausser and D. Stehlik, Adv. Magn. Reson. 79 (1968).

${ }^{23}$ I. Solomon, Phys. Rev. 99, 559 (1955).

${ }^{24}$ M. Bennati, C. Luchinat, G. Parigi, and M.-T. Turke, Phys. Chem. Chem. Phys. 12, 5902 (2010).

${ }^{25}$ T. Orlando, R. Dervişoğlu, M. Levien, I. Tkach, T.F. Prisner, L.B. Andreas, V.P. Denysenkov, and M. 
This article may be downloaded for personal use only. Any other use requires prior permission of the author and AIP Publishing. This article appeared in J. Chem. Phys. 152, 034202 (2020) and may be found at https://doi.org/10.1063/1.5133408

Bennati, Angew. Chemie - Int. Ed. 58, 1402 (2019).

${ }^{26}$ G. Liu, M. Levien, N. Karschin, G. Parigi, C. Luchinat, and M. Bennati, Nat. Chem. 9, 676 (2017).

${ }^{27}$ D.J. Cheney and C.J. Wedge, Electron Paramagn. Reson. 26, 89 (2019).

${ }^{28}$ M.D.E. Forbes, L.E. Jarocha, S. Sim, and V.F. Tarasov, Adv. Phys. Org. Chem. 47, 1 (2013).

${ }^{29}$ T. Imamura and K. Obi, J Phys Chem 90, 6741 (1986).

${ }^{30}$ C. Blättler, F. Jent, and H. Paul, Chem. Phys. Lett. 166, 375 (1990).

${ }^{31}$ A. Kawai and K. Shibuya, J. Photochem. Photobiol. C Photochem. Rev. 7, 89 (2006).

${ }^{32}$ A.I. Shushin, Chem. Phys. Lett. 208, 173 (1993).

${ }^{33}$ L. Ludvíková, P. Fris, D. Heger, P. Sebej, J. Wirz, and P. Klan, Phys. Chem. Chem. Phys. 18, 16266 (2016).

${ }^{34}$ C. Lambert, T. Sarna, and T.G. Truscott, J. Chem. Soc. Faraday Trans. 86, 3879 (1990).

${ }^{35}$ V. Wintgens, J.C. Scaiano, S.M. Linden, and D.C. Neckers, J. Org. Chem. 54, 5242 (1989).

${ }^{36}$ C.R. Lambert and I.E. Kochevar, Photochem. Photobiol. 66, 15 (1997).

${ }^{37}$ M.W. Dale, C. Vallotto, and C.J. Wedge, Prep. (2019).

${ }^{38}$ A. Blank and H. Levanon, J. Phys. Chem. A 104, 794 (2000).

${ }^{39}$ L. Hwang and J. Freed, J. Chem. Phys. 63, 4017 (1975).

${ }^{40}$ B.D. Armstrong and S. Han, J. Am. Chem. Soc. 131, 4641 (2009).

${ }^{41}$ J.R. Biller, H. Elajaili, V. Meyer, G.M. Rosen, S.S. Eaton, and G.R. Eaton, 236, 47 (2013).

${ }^{42}$ J.H. Lee and S. Cavagnero, J. Phys. Chem. B 117, 6069 (2013). 


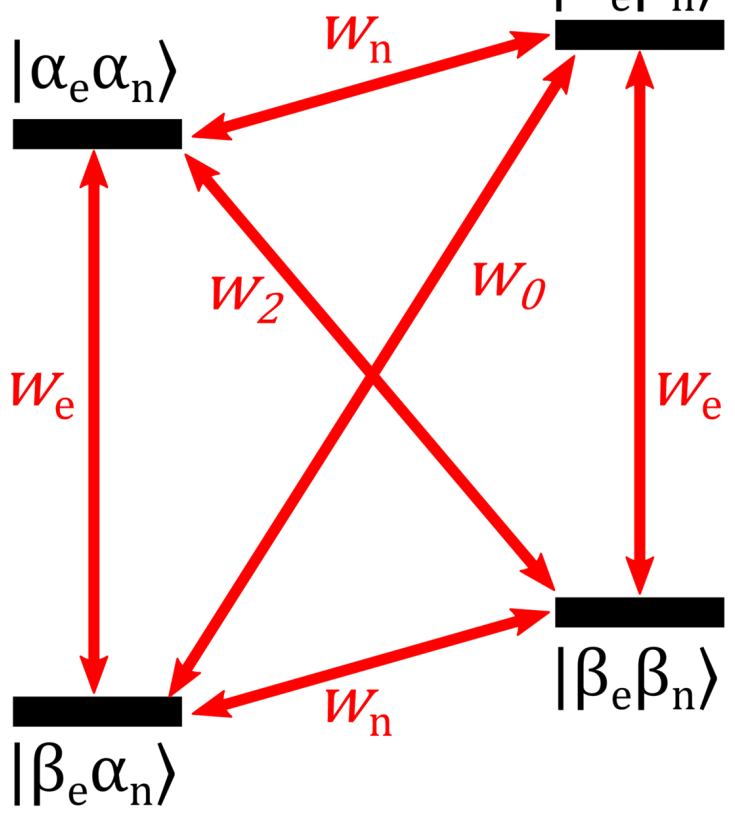


(a)

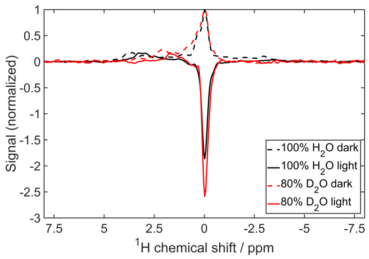

(c)

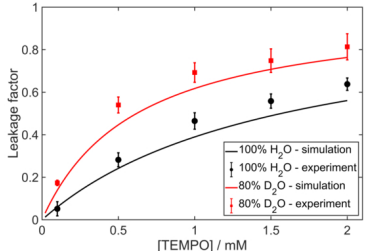

(b)

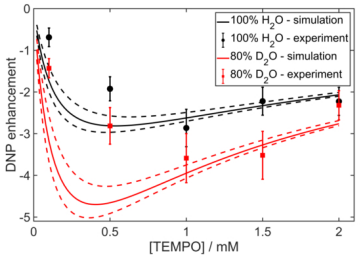

(d)

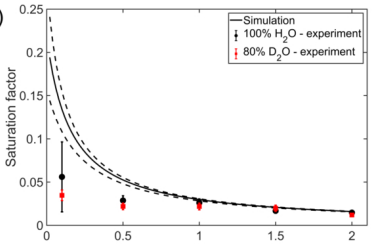



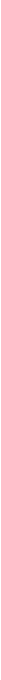
(a)

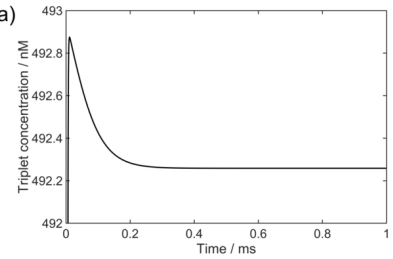

(c)

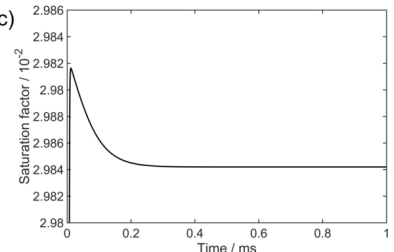

(b)

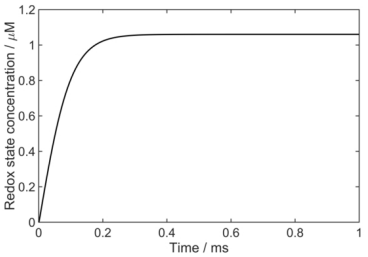

(d)

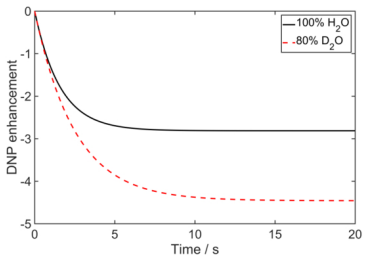


(a)

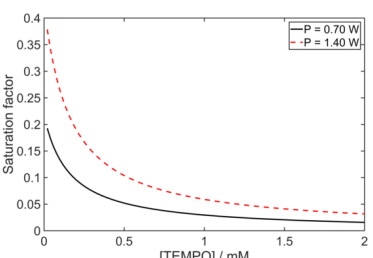

(b)

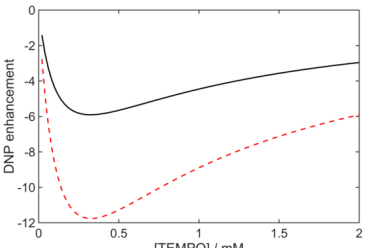


(a)

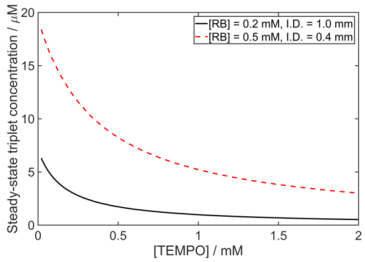

(b)

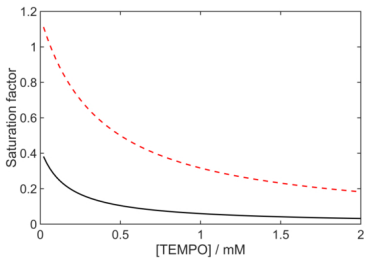

(c)

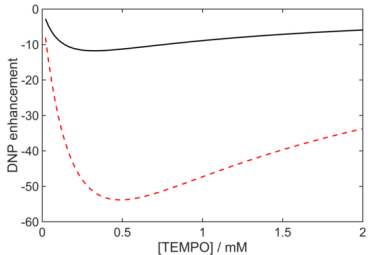


(a)

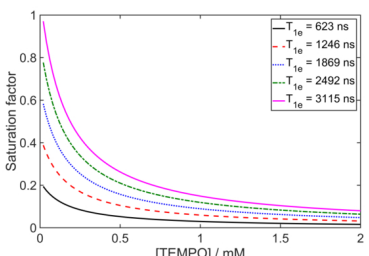

(b)

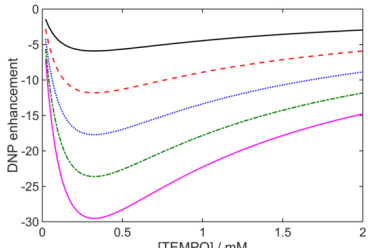


(a)

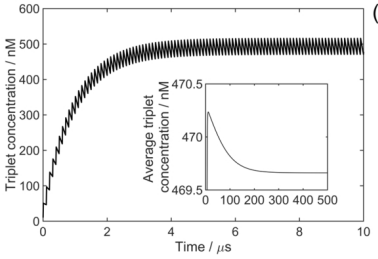

(b)

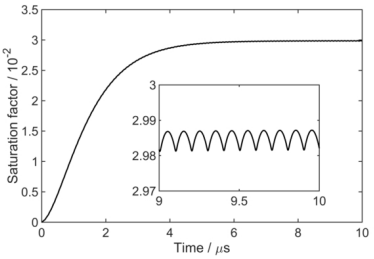

(c)

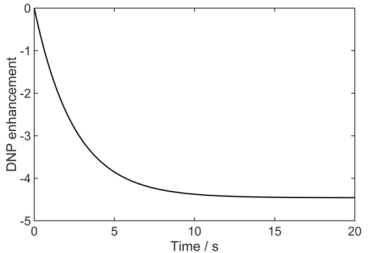


(a)

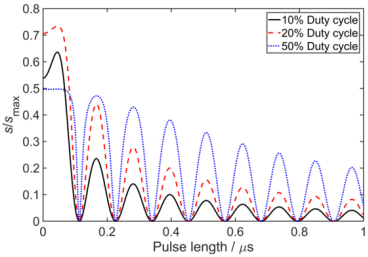

(b)

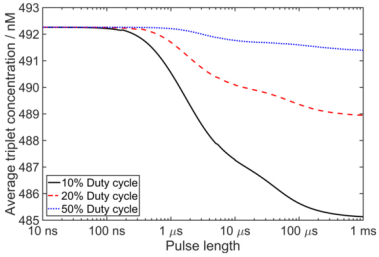

(c)

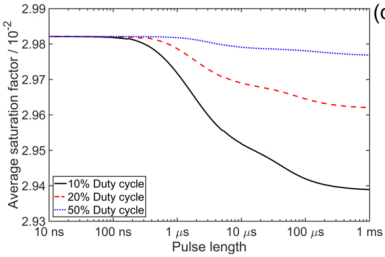

(d)

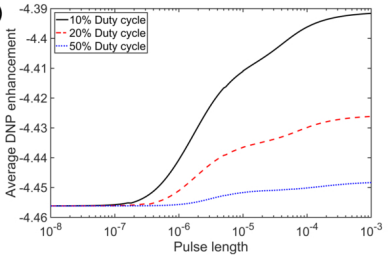

(e)
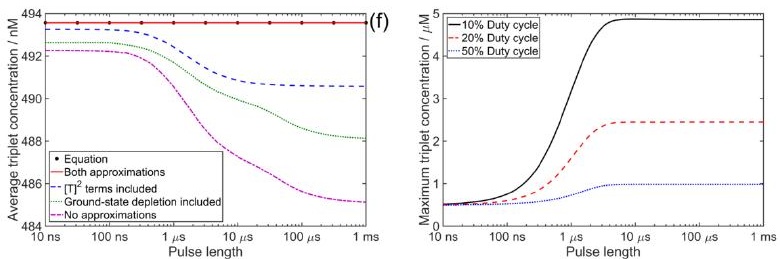
(a)

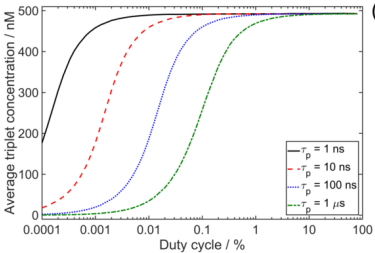

(c)

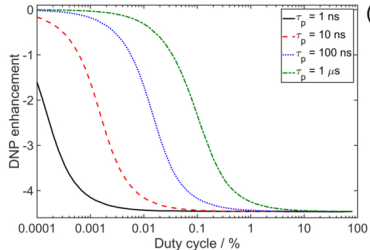

(b)

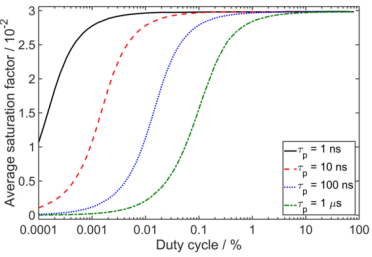

(d)

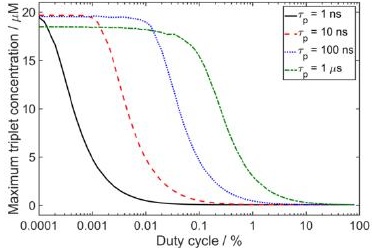

